\title{
A CLASS OF DISCRETE PREDATOR-PREY INTERACTION WITH BIFURCATION ANALYSIS AND CHAOS CONTROL
}

\author{
Qamar Din $^{1, *}$, Nafeesa Saleem ${ }^{1}$ and Muhammad SajJad Shabbir ${ }^{2}$
}

\begin{abstract}
The interaction between prey and predator is well-known within natural ecosystems. Due to their multifariousness and strong link population dynamics, predators contain distinct features of ecological communities. Keeping in view the Nicholson-Bailey framework for host-parasitoid interaction, a discrete-time predator-prey system is formulated and studied with implementation of type-II functional response and logistic prey growth in form of the Beverton-Holt map. Persistence of solutions and existence of equilibria are discussed. Moreover, stability analysis of equilibria is carried out for predator-prey model. With implementation of bifurcation theory of normal forms and center manifold theorem, it is proved that system undergoes transcritical bifurcation around its boundary equilibrium. On the other hand, if growth rate of consumers is taken as bifurcation parameter, then system undergoes Neimark-Sacker bifurcation around its positive equilibrium point. Methods of chaos control are introduced to avoid the populations from unpredictable behavior. Numerical simulation is provided to strengthen our theoretical discussion.
\end{abstract}

Mathematics Subject Classification. 39A30, 40A05, 92D25, 92C50.

Received March 10, 2020. Accepted September 11, 2020.

\section{INTRODUCTION}

Many natural ecosystems in our universe are related to host-parasitoid interaction and most of the hosts and their parasitoids have life cycles of synchronized form. Therefore, difference equations are more appropriate for dynamical study of such interactions. Oscillations with infinitely increasing amplitude are observed in case of classical Nicholson-Bailey (NB) model [3]. On the other hand, the natural host-parasitoid interactions never show such oscillatory behavior. Therefore, classical NB model is not a good representative of natural hostparasitoid interaction due to instability of coexistence and its oscillatory behavior. Some additional ecological process is needed for stability of coexistence. Many modifications of the NB model, particularly introducing density dependence effects $[6,37,62]$, enabled this coexistence to occur. For some other notable modifications in host-parasitoid interaction, we refer to $[4,5,23,25,26,30,31,34,36,48,50,58,59]$ and references therein.

The present discussion is based on framework of classical NB model to formulate a class of predator-prey interaction for non-overlapping generations. It is one of the most plain discrete-time prey-predator models, and it has demonstrated an outstanding role in the search for stabilizing mechanisms in host-parasitoid and predatorprey interactions [22]. The dynamical relation between prey and predators is a significant topic in environmental

Keywords and phrases: Predator-prey model, stability, transcritical bifurcation, Neimark-Sacker bifurcation, chaos control.

${ }^{1}$ Department of Mathematics, University of Poonch Rawalakot, Azad Kashmir, Pakistan.

2 Department of Mathematics, Air University, Islamabad, Pakistan.

* Corresponding author: qamar.sms@gmail.com

(C) The authors. Published by EDP Sciences, 2020 
science and has been examined in many mathematical models (cf. $[2,27,38,51])$. Mathematical models can be expressed in continuous time (for overlapping generations) and discrete time (for non-overlapping generations). Continuous-time dynamical systems have been used over a great extent to investigate the qualitative behavior of interacting species for many years $($ see $[8,21])$. On the other hand, there are many models with dynamics which are suitably described by discrete time, for example, species with seasonal breeding and yearly life cycle species [7]. Furthermore, a suitable justification for analyzing discrete-time models is that data are collected at discrete times and hence it may be easier to compare data with the output of a discrete model [9].

Discretization of continuous-time dynamical systems is one of the method to obtain discrete-time dynamical systems governed by difference equations. Some numerical techniques have been frequently used to obtain discrete counterparts predator - prey systems. On the other hand, a discrete dynamical system is called dynamically consistent with its continuous counterpart if both display an identical dynamical behavior, such as persistence and boundedness of solutions, local and global behaviors of fixed points, bifurcation, and chaotic behavior [32]. Piecewise constant arguments or exponential-discretization (see [18, 24]) and Euler approximations (see $[33,39,55])$ are more frequently used methods to obtain discrete-time counterparts of predator-prey models. But both of these methods are lacking the dynamical consistency with their continuous counterparts.

On the other hand, Rosenzweig-MacArthur [46] is one of the simplest continuous models in population biology to present a Hopf bifurcation. Furthermore, Rosenzweig-MacArthur model also reveals paradox of enrichment and hydra effect. A discrete counterpart of Rosenzweig-MacArthur model was studied by Hadeler and Gerstmann [20] with implementation of Euler approximation. But this discrete counterpart of RosenzweigMacArthur model is not consistency and bifurcation preserving. Recently, Weide et al. [56] proposed some discrete prey-predator models by adopting NB framework and implementation of type-II functional response and logistic prey growth in form of the Beverton-Holt map. These models reveal behaviors, that is, paradox of enrichment, hydra effect and Neimark-Sacker bifurcation, similar to Rosenzweig-MacArthur model.

Arguing as in [56], we consider the following modification of NB model:

$$
\begin{aligned}
& x_{n+1}=x_{n} G\left(x_{n}, y_{n}\right) F\left(x_{n} G\left(x_{n}, y_{n}\right)\right), \\
& y_{n+1}=b x_{n}\left(1-G\left(x_{n}, y_{n}\right)\right), n=0,1,2, \ldots,
\end{aligned}
$$

where $x_{n}$ and $y_{n}$ are population densities of prey and predator, respectively, at generation $n ; b$ is the conversion efficiency from prey to predator, and $F(x)$ is Beverton-Holt growth function defined by

$$
F(x)=\frac{\lambda}{1+\frac{\lambda-1}{k} x},
$$

where $k>0$ is the carrying capacity and $\lambda>1$ is intrinsic per-capita growth. Moreover, $G(x, y)$ is type-II functional response given by

$$
G(x, y)=\exp \left(-\frac{a y}{1+h x}\right)
$$

where $a$ denotes predation rate and $h$ represents handling time of predators per prey. Putting the values of $F$ and $G$ in system (1.1), we obtain the following predator-prey system:

$$
\begin{aligned}
& x_{n+1}=\frac{\lambda x_{n} \exp \left(-\frac{a y_{n}}{1+h x_{n}}\right)}{1+\frac{\lambda-1}{k} x_{n} \exp \left(-\frac{a y_{n}}{1+h x_{n}}\right)}, \\
& y_{n+1}=b x_{n}\left(1-\exp \left(-\frac{a y_{n}}{1+h x_{n}}\right)\right) .
\end{aligned}
$$


TABLE 1. Variables and parameters used in system (1.2) with their description and units.

\begin{tabular}{ccc}
\hline Parameter/Variable & Biological meaning & Unit \\
\hline$x_{n}$ & Biomass density of prey & Biomass per unit area \\
$y_{n}$ & Biomass density of predator & Biomass per unit area \\
$k$ & Carrying capacity of environment for prey & Biomass per unit area \\
$\lambda$ & Intrinsic per-capita growth rate of prey & Per day \\
$a$ & Predation rate & Per day \\
$h$ & Predator handling time & Day \\
$b$ & Conversion efficiency from prey to predator & Dimensionless \\
\hline
\end{tabular}

Furthermore, biological meanings and units of state variables and parametric values of system (1.2) are presented in Table 1. We summarize the remaining discussion of this paper as follows. Persistence of solutions for system (1.2) is discussed in Section 2. Section 3 is dedicated to existence of biologically feasible equilibria, and conditions for local asymptotic stability of these equilibria are also investigated. In Section 4, we show that boundary equilibrium of system (1.2) undergoes transcritical bifurcation whenever growth parameter $b$ of predator population is taken as bifurcation parameter. In Section 5, it is proved that system (1.2) undergoes Neimark-Sacker bifurcation around its interior equilibrium point. OGY, hybrid control and exponential-type control methods are introduced in Section 6. Lastly, numerical simulations are provided in Section ?? to illustrate our theoretical discussion.

\section{BOUNDEDNESS AND PERSISTENCE}

In this section, we show that every solution of system (1.2) persists under certain parametric conditions. For this, the following Theorem is presented.

Theorem 2.1. Assume that $\lambda>e^{a b k}$ and $a b\left(\lambda-e^{a b k}\right)>1+h k$, then every solution of (1.2) is persistence.

Proof. See Appendix A.

With mathematical induction one can prove the following result.

Lemma 2.2. Assume that $0<x_{0} \leq k$ and $0<y_{0} \leq b k$, then the rectangle $[0, k] \times[0, b k]$ is invariant interval for every positive solution $\left\{\left(x_{n}, y_{n}\right)\right\}$ of the system (1.2).

\section{EXISTENCE OF EQUILIBRIA AND STABILITY ANALYSIS}

In this section, first we explore the biologically feasible fixed points of system (1.2). The steady-states of system (1.2) satisfy the following two-dimensional algebraic equations:

$$
\begin{aligned}
& x=\frac{\lambda x \exp \left(-\frac{a y}{1+h x}\right)}{1+\frac{\lambda-1}{k} x \exp \left(-\frac{a y}{1+h x}\right)}, \\
& y=b x\left(1-\exp \left(-\frac{a y}{1+h x}\right)\right) .
\end{aligned}
$$

Then, it is quite easy to see that $O=(0,0)$ and $E=(k, 0)$ are trivial and boundary equilibria of $(1.2)$, respectively. We are interested in interior equilibrium, which cannot be investigated in closed form. Neglecting the 
trivial solution of system (3.1), we left with the following algebraic system for coexistence:

$$
\begin{aligned}
& \exp \left(\frac{a y}{1+h x}\right)+\frac{\lambda-1}{k} x-\lambda=0, \\
& y-b x\left(1-\exp \left(-\frac{a y}{1+h x}\right)\right)=0 .
\end{aligned}
$$

From first equation of system (3.2), it follows that

$$
\exp \left(\frac{a y}{1+h x}\right)=\lambda-\frac{\lambda-1}{k} x
$$

Solving (3.3) for $y$ gives

$$
y=\frac{1+h x}{a} \ln \left(\lambda-\frac{\lambda-1}{k} x\right) .
$$

Similarly, from second equation of system (3.2) and (3.3), it follows that

$$
y=b x\left(1-\frac{1}{\lambda-\frac{(\lambda-1) x}{k}}\right) .
$$

Comparison of (3.4) and (3.5) with simplification yields that:

$$
\frac{a b x\left(1-\frac{1}{\lambda-\frac{(\lambda-1) x}{k}}\right)}{h x+1}=\ln \left(\lambda-\frac{\lambda-1}{k} x\right) .
$$

From (3.6), it follows that $x^{*}$ is a positive real root for the following transcendental equation:

$$
\Psi_{1}\left(x^{*}\right)=\Psi_{2}\left(x^{*}\right)
$$

where

$$
\Psi_{1}(x):=\frac{a b x\left(1-\frac{1}{\lambda-\frac{(\lambda-1) x}{k}}\right)}{h x+1},
$$

and

$$
\Psi_{2}(x):=\ln \left(\lambda-\frac{\lambda-1}{k} x\right)
$$

Simple calculation yields that $\Psi_{2}(0)=\ln (\lambda)>0, \Psi_{2}(k)=0$ and $\Psi_{1}(0)=\Psi_{1}(k)=0$. On the other hand, $\Psi_{2}^{\prime}(x)=-\frac{\lambda-1}{k \lambda-\lambda x+x}<0$ and $\Psi_{1}^{\prime \prime}(x)<0$ for all $x \in(0, k)$. Therefore, $\Psi_{2}(x)$ is monotonically decreasing and $\Psi_{1}(x)$ is concave down in $(0, k)$. Hence, transcendental equation (3.7) has a unique solution $x^{*}$ satisfying $0<x^{*}<k$.

For $a=1.1, b=1.5, \lambda=2.9, h=0.65$ and $k=19.3$, the intersection of $\Psi_{1}(x)$ and $\Psi_{2}(x)$ is depicted in Figure 1. Now, the following Lemma gives the dynamics of trivial and boundary equilibria of system (1.2). 


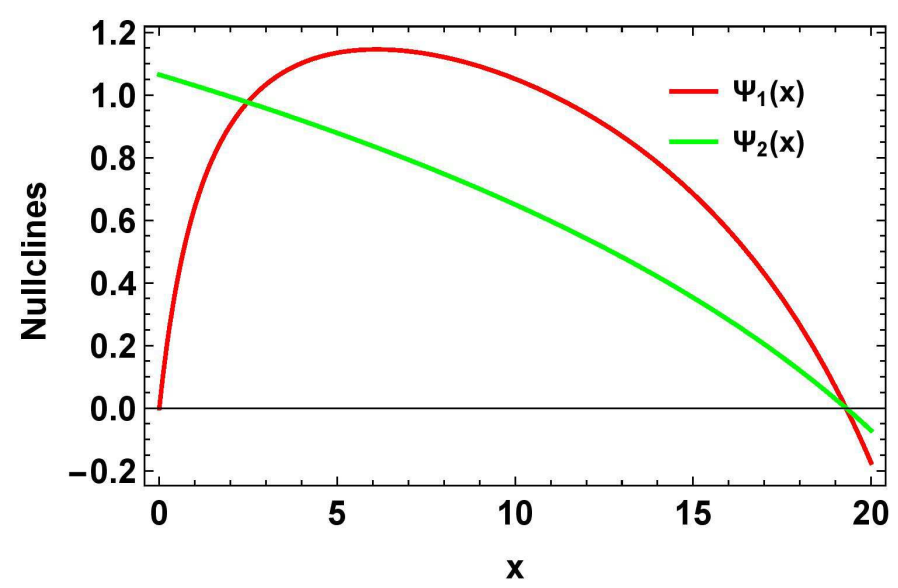

Figure 1. Coexistence of system (1.2) with $a=1.1, b=1.5, \lambda=2.9, h=0.65$ and $k=19.3$.

Lemma 3.1. The following results hold true for system (1.2):

(i) The trivial equilibrium $O=(0,0)$ is a saddle point.

(ii) The boundary equilibrium $E=(k, 0)$ is a sink if and only if $\frac{a b k}{h k+1}<1$, it is a saddle point if and only if $\frac{a b k}{h k+1}>1$ and $E$ is a non-hyperbolic equilibrium point if and only if $\frac{a b k}{h k+1}=1$.

Proof. (i) First, it is easy to see that the Jacobian matrix of (1.2) at trivial equilibrium $O$ is given by:

$$
J(O)=\left(\begin{array}{ll}
\lambda & 0 \\
0 & 0
\end{array}\right)
$$

Then, eigenvalues of $J(O)$ are $\mu_{1}=\lambda>1$ and $\mu_{2}=0<1$. Therefore, $O$ is a saddle point.

(ii) Secondly, it is easy to see that the Jacobian matrix of (1.2) at boundary equilibrium $E$ is given by:

$$
J(E)=\left(\begin{array}{cc}
\frac{1}{\lambda} & -\frac{a k}{h k \lambda+\lambda} \\
0 & \frac{a b k}{h k+1}
\end{array}\right)
$$

Then, eigenvalues of $J(O)$ are $\mu_{1}=\frac{1}{\lambda}<1$ and $\mu_{2}=\frac{a b k}{h k+1}$. Therefore, it follows that $E=(k, 0)$ is a sink if and only if $\frac{a b k}{h k+1}<1$, it is a saddle point if and only if $\frac{a b k}{h k+1}>1$ and $E$ is a non-hyperbolic equilibrium point if and only if $\frac{a b k}{h k+1}=1$.

Moreover, system (1.2) undergoes transcritical bifurcation at its boundary equilibrium $E=(k, 0)$ whenever the parameter $b$ varies in a small neighborhood of $b \equiv b_{0}=\frac{h k+1}{a k}$. Classification of $E$ with transcritical bifurcation curve (yellow curve) in $a b$-plane is depicted in Figure 2. Next, we see dynamics of system (1.2) at its positive steady-state, say, $\left(x^{*}, y^{*}\right)$, which satisfies the following algebraic system:

$$
\begin{aligned}
& \exp \left(\frac{a y^{*}}{1+h x^{*}}\right)+\frac{\lambda-1}{k} x^{*}-\lambda=0, \\
& y^{*}-b x^{*}\left(1-\exp \left(-\frac{a y^{*}}{1+h x^{*}}\right)\right)=0 .
\end{aligned}
$$




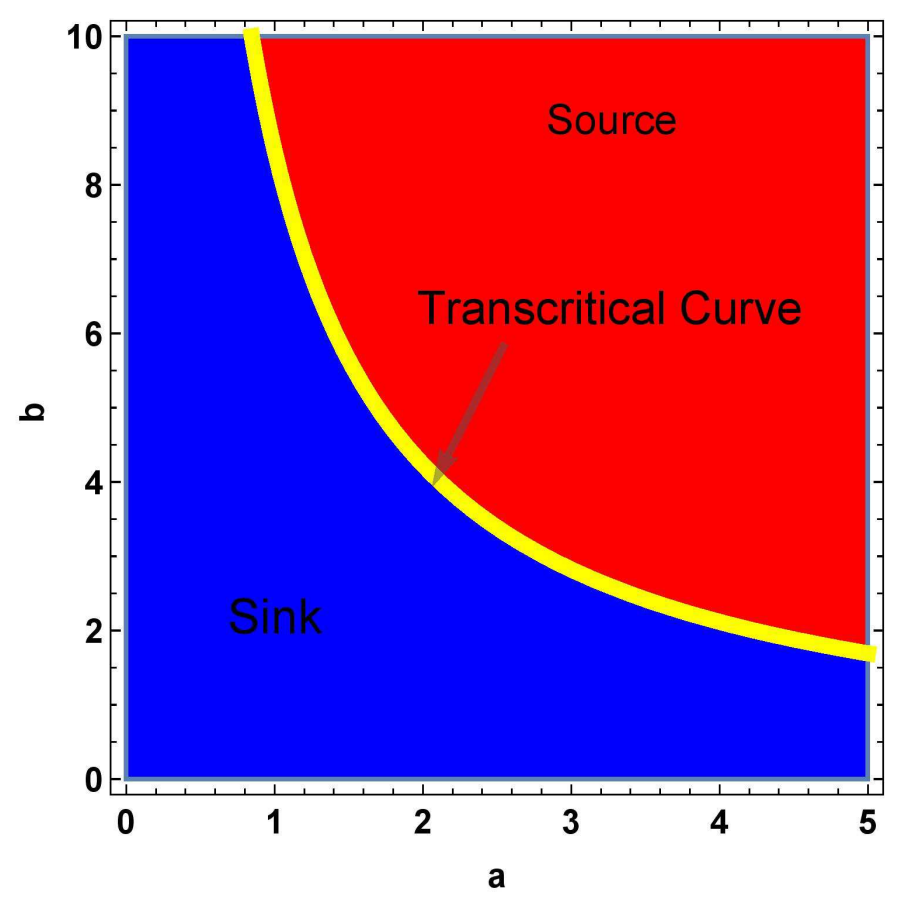

Figure 2. Classification of $E$ with $h=7.8$ and $k=1.5$.

The Jacobian matrix $J\left(x^{*}, y^{*}\right)$ of system (1.2) evaluated at $\left(x^{*}, y^{*}\right)$ is given by:

$$
J\left(x^{*}, y^{*}\right)=\left(\begin{array}{cc}
\frac{e^{\frac{a y^{*}}{h x^{*}+1}}\left(a h x^{*} y^{*}+\left(h x^{*}+1\right)^{2}\right)}{\lambda\left(h x^{*}+1\right)^{2}} & -\frac{a x^{*} e^{\frac{a y^{*}}{h x^{*}+1}}}{\lambda\left(h x^{*}+1\right)} \\
\frac{y^{*}}{x^{*}}-\frac{a b h x^{*} y^{*} e^{-\frac{a y^{*}}{h x^{*}+1}}}{\left(h x^{*}+1\right)^{2}} & \frac{a b x^{*} e^{-\frac{a y^{*}}{h x^{*}+1}}}{h x^{*}+1}
\end{array}\right)
$$

Now characteristic polynomial of $J\left(x^{*}, y^{*}\right)$ is given by:

$$
P(\mu)=\mu^{2}-\left(\frac{e^{\frac{a y^{*}}{h x^{*}+1}}\left(a h x^{*} y^{*}+\left(h x^{*}+1\right)^{2}\right)}{\lambda\left(h x^{*}+1\right)^{2}}+\frac{a b x^{*} e^{-\frac{a y^{*}}{h x^{*}+1}}}{h x^{*}+1}\right) \mu+\frac{a\left(y^{*} e^{\frac{a y^{*}}{h x^{*}+1}}+b x^{*}\right)}{h \lambda x^{*}+\lambda} .
$$

The following Lemma gives dynamical behavior of system (1.2) at its positive fixed point.

Lemma 3.2. The following results hold true for system (1.2):

(i) The positive equilibrium $\left(x^{*}, y^{*}\right)$ is a sink if and only if

$$
a b \lambda x^{*}\left(h x^{*}+1\right)-\left(h x^{*}+1\right) e^{\frac{a y^{*}}{h x^{*}+1}}\left(a b x^{*}+h \lambda x^{*}+\lambda\right)+e^{\frac{2 a y^{*}}{h x^{*}+1}}\left(\left(h x^{*}+1\right)^{2}-a y^{*}\right)<0,
$$

and

$$
\frac{a\left(y^{*} e^{\frac{a y^{*}}{h x^{*}+1}}+b x^{*}\right)}{h \lambda x^{*}+\lambda}<1
$$




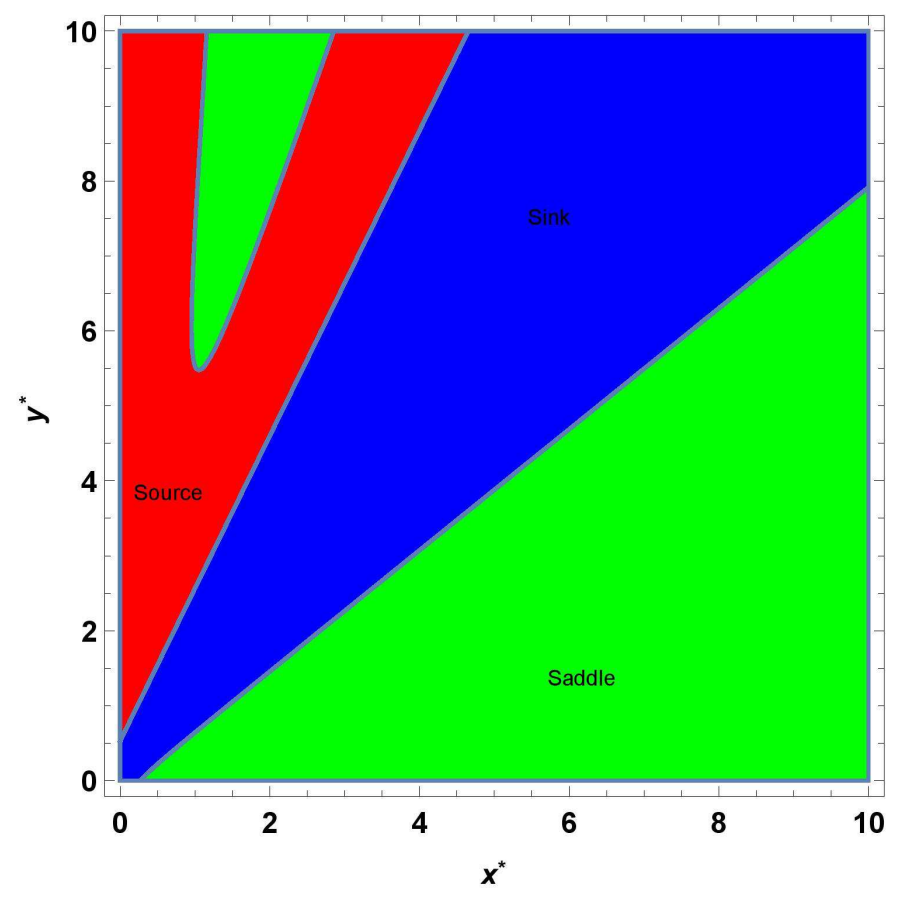

Figure 3. Classification of $\left(x^{*}, y^{*}\right)$ with $a=3.3, b=2.4, h=4.2$ and $\lambda=9.8$.

(ii) The positive equilibrium $\left(x^{*}, y^{*}\right)$ is a saddle point if and only if

$$
a b \lambda x^{*}\left(h x^{*}+1\right)-\left(h x^{*}+1\right) e^{\frac{a y^{*}}{h x^{*}+1}}\left(a b x^{*}+h \lambda x^{*}+\lambda\right)+e^{\frac{2 a y^{*}}{h x^{*}+1}}\left(\left(h x^{*}+1\right)^{2}-a y^{*}\right)>0 .
$$

(iii) The positive equilibrium $\left(x^{*}, y^{*}\right)$ is a source if and only if

$$
a b \lambda x^{*}\left(h x^{*}+1\right)-\left(h x^{*}+1\right) e^{\frac{a y^{*}}{h x^{*}+1}}\left(a b x^{*}+h \lambda x^{*}+\lambda\right)+e^{\frac{2 a y^{*}}{h x^{*}+1}}\left(\left(h x^{*}+1\right)^{2}-a y^{*}\right)<0,
$$

and

$$
\frac{a\left(y^{*} e^{\frac{a y^{*}}{h x^{*}+1}}+b x^{*}\right)}{h \lambda x^{*}+\lambda}>1 .
$$

Next, it is interesting to see the dynamical classification of system (1.2) in $x^{*} y^{*}$-plane about its positive fixed point. For such classification, various parametric values are selected for system (1.2). Moreover, regions for sink, source and saddle cases are represented by blue, red and green regions, respectively. For $a=3.3, b=2.4$, $h=4.2$ and $\lambda=9.8$ dynamical classification is depicted in Figure 3. On the other hand, for comparatively smaller parametric values, that is, $a=0.36, b=0.5, h=0.42$ and $\lambda=1.4$, the dynamical classification is depicted in Figure 4. 


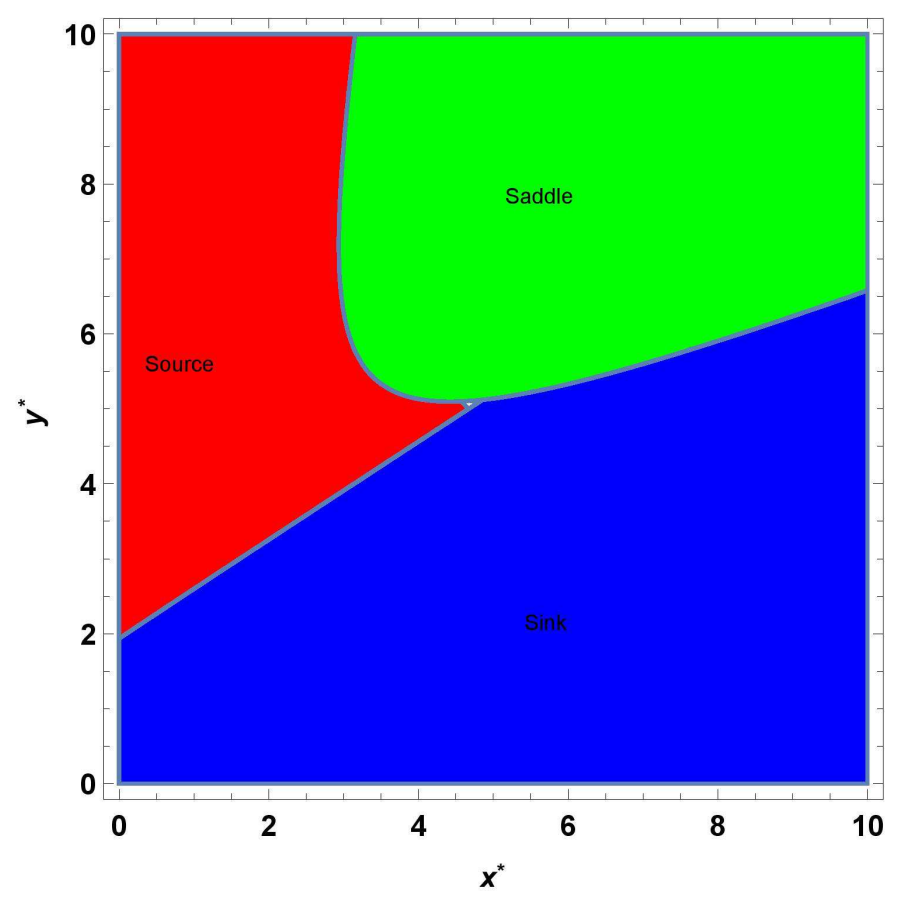

Figure 4. Classification of $\left(x^{*}, y^{*}\right)$ with $a=0.36, b=0.5, h=0.42$ and $\lambda=1.4$.

\section{TRANSCRITICAL BIFURCATION}

In this section, we apply bifurcation theory of normal forms and center manifold theorem for emergence of transcritical bifurcation around boundary equilibrium of system (1.2). For this, first we assume that

$$
b \equiv b_{0}=\frac{h k+1}{a k} .
$$

Moreover, we consider the following curve

$$
C_{T B}:=\left\{(a, b, k, h, \lambda) \in \mathbb{R}_{+}^{5}: b \equiv b_{0}=\frac{h k+1}{a k}, \lambda>1\right\} .
$$

Next, we suppose that $(a, b, k, h, \lambda) \in C_{T B}$, and writing system (1.2) in the following equivalent map:

$$
\left(\begin{array}{l}
u \\
v
\end{array}\right) \rightarrow\left(\begin{array}{c}
\frac{\lambda u \exp \left(-\frac{a v}{1+h u}\right)}{1+\frac{\lambda-1}{k} u \exp \left(-\frac{a v}{1+h u}\right)} \\
\left(b_{0}+\hat{b}\right) u\left(1-\exp \left(-\frac{a v}{1+h u}\right)\right)
\end{array}\right)
$$

where $\hat{b}$ is very small perturbation in $b_{0}$. Moreover, taking into account the translations $p=u-k$ and $q=v$, then map (4.1) is converted into the following map with fixed point at origin:

$$
\left(\begin{array}{c}
p \\
q
\end{array}\right) \rightarrow\left(\begin{array}{cc}
\frac{1}{\lambda} & -\frac{a k}{h k \lambda+\lambda} \\
0 & \frac{a b_{0} k}{h k+1}
\end{array}\right)\left(\begin{array}{c}
p \\
q
\end{array}\right)+\left(\begin{array}{c}
\phi(p, q) \\
\psi(p, q, \hat{b})
\end{array}\right)
$$


where

$$
\begin{aligned}
& \phi(p, q)=m_{13} p^{2}+m_{14} p q+m_{15} q^{2}+m_{16} p^{3}+m_{17} p^{2} q+m_{18} p q^{2}+m_{19} q^{3}+O\left((|p|+|q|)^{4}\right), \\
& \psi(p, q, \hat{b})=m_{23} p q+m_{24} q^{2}+m_{25} p^{2} q+m_{26} p q^{2}+m_{27} q^{3}+c_{1} q \hat{b}+c_{2} q^{2} \hat{b} \\
& +c_{3} p q \hat{b}+O\left((|p|+|q|+|\hat{b}|)^{4}\right) \text {, } \\
& m_{13}=\frac{1-\lambda}{k \lambda^{2}}, m_{14}=\frac{a(2 h k(\lambda-1)+\lambda-2)}{(h k \lambda+\lambda)^{2}}, m_{15}=-\frac{a^{2} k(\lambda-2)}{2(h k \lambda+\lambda)^{2}}, m_{16}=\frac{(\lambda-1)^{2}}{k^{2} \lambda^{3}}, \\
& m_{17}=-\frac{a\left(3 h^{2} k^{2}(\lambda-1)^{2}+h k(\lambda(3 \lambda-10)+6)+\lambda^{2}-4 \lambda+3\right)}{k(h k \lambda+\lambda)^{3}}, \\
& m_{18}=\frac{a^{2}(h k(\lambda(3 \lambda-10)+6)+(\lambda-6) \lambda+6)}{2(h k \lambda+\lambda)^{3}}, m_{19}=-\frac{a^{3} k((\lambda-6) \lambda+6)}{6(h k \lambda+\lambda)^{3}}, \\
& m_{23}=\frac{a b_{0}}{(h k+1)^{2}}, m_{24}=-\frac{a^{2} b_{0} k}{2(h k+1)^{2}}, m_{25}=-\frac{a b_{0} h}{(h k+1)^{3}}, m_{26}=\frac{a^{2} b_{0}(h k-1)}{2(h k+1)^{3}}, \\
& m_{27}=\frac{a^{3} b_{0} k}{6(h k+1)^{3}}, c_{1}=\frac{a k}{h k+1}, c_{2}=-\frac{a^{2} k}{2(h k+1)^{2}}, c_{3}=\frac{a}{(h k+1)^{2}} .
\end{aligned}
$$

Considering the following transformation

$$
\left(\begin{array}{c}
p \\
q
\end{array}\right)=T\left(\begin{array}{l}
x \\
y
\end{array}\right)
$$

where

$$
T=\left(\begin{array}{cc}
-\frac{a k}{a b_{0} \lambda k-h k-1} & 1 \\
1 & 0
\end{array}\right)
$$

From (4.2) and (4.3), we obtain

$$
\left(\begin{array}{c}
x \\
y
\end{array}\right) \rightarrow\left(\begin{array}{cc}
1 & 0 \\
0 & \frac{1}{\lambda}
\end{array}\right)\left(\begin{array}{l}
x \\
y
\end{array}\right)+\left(\begin{array}{l}
\phi_{1}(x, y, \hat{b}) \\
\psi_{1}(x, y, \hat{b})
\end{array}\right)
$$

where

$$
\begin{aligned}
\phi_{1}(x, y, \hat{b})= & m_{25} p^{2} q+m_{26} p q^{2}+\left(\hat{b} c_{3}+m_{23}\right) p q+m_{27} q^{3}+\left(\hat{b} c_{2}+m_{24}\right) q^{2} \\
& +c_{1} q \hat{b}+O\left((|x|+|y|+|\hat{b}|)^{4}\right)
\end{aligned}
$$




$$
\begin{aligned}
\psi_{1}(x, y, \hat{b})= & m_{16} p^{3}+\left(\delta m_{25}+m_{17}\right) p^{2} q+m_{13} p^{2}+\left(\delta m_{26}+m_{18}\right) q^{2} p \\
& +\left(m_{14}+\delta\left(\hat{b} c_{3}+m_{23}\right)\right) p q+\left(\delta m_{27}+m_{19}\right) q^{3}+\left(m_{15}+\delta\left(\hat{b} c_{2}+m_{24}\right)\right) q^{2} \\
+ & \delta \hat{b} c_{1} q+O\left((|x|+|y|+|\hat{b}|)^{4}\right), \\
& p=y-\delta x, q=x \text { and } \delta=\frac{a k}{a b_{0} k \lambda-h k-1} .
\end{aligned}
$$

Due to center manifold theory [10], stability analysis of equilibrium $(x, y)=(0,0)$ near $\hat{b}=0$ can be discussed by investigating a one-parameter family of reduced equations on a center manifold $W^{C}(0,0,0)$, which can be described as follows:

$$
W^{C}(0,0,0)=\left\{(x, y, \hat{b}) \in \mathbb{R}^{3}: y=l_{1} x^{2}+l_{2} x \hat{b}+l_{3} \hat{b}^{2}+O\left((|x|+|\hat{b}|)^{3}\right)\right\},
$$

where

$$
l_{1}=\frac{\left(m_{13} \delta^{2}-\left(\delta m_{23}+m_{14}\right) \delta+\delta m_{24}+m_{15}\right)}{\left(1-\lambda^{-1}\right)}, l_{2}=\frac{\delta c_{1}}{\left(1-\lambda^{-1}\right)}, l_{3}=0 .
$$

On the other hand, the map restricted to center manifold is described as follows:

$$
F: x \rightarrow x+\hat{b}+k_{1} x^{2}+k_{2} x \hat{b}+k_{3} \hat{b}^{2}+O\left((|x|+|\hat{b}|)^{3}\right)
$$

where

$$
k_{1}=m_{13} \delta^{2}-\left(\delta m_{23}+m_{14}\right) \delta+\delta m_{24}+m_{15}, k_{2}=\delta c_{1}, k_{3}=0
$$

Then, it is easy to see that

$$
F(0,0)=0, F_{x}(0,0)=1, F_{\hat{b}}(0,0)=1,
$$

and

$$
\kappa:=F_{x x}(0,0)=2 m_{13} \delta^{2}-2\left(\delta m_{23}+m_{14}\right) \delta+2 \delta m_{24}+2 m_{15} .
$$

The following Theorem gives conditions for emergence of transcritical bifurcation about boundary equilibrium of system (1.2).

Theorem 4.1. Assume that $\kappa \neq 0$ and $b=\frac{h k+1}{a k}$, then system (1.2) undergoes transcritical bifurcation around its boundary equilibrium when parameter $b$ changes in small neighborhood of $b_{0}=\frac{h k+1}{a k}$. Moreover, two fixed points bifurcate from boundary equilibrium for $b<b_{0}$, and merge as the boundary equilibrium at $b=b_{0}$ and disappear at $b>b_{0}$.

\section{NEIMARK-SACKER BIFURCATION}

In this section, we discuss that system (1.2) undergoes Neimark-Sacker bifurcation around its positive equilibrium $\left(x^{*}, y^{*}\right)$. For this, $b$ is taken as bifurcation parameter. For emergence of Neimark-Sacker bifurcation around positive equilibrium $\left(x^{*}, y^{*}\right)$ of system (1.2), the roots of (3.8) must be complex conjugate with unit 
modulus. Therefore, necessary conditions for emergence of Neimark-Sacker about positive fixed point of system (1.2) are described by the following curve:

$$
C_{N B}=\left\{(a, b, k, h, \lambda) \in \mathbb{R}_{+}^{5}: b \equiv b_{1}=\frac{h \lambda x^{*}+\lambda-a y^{*} e^{\frac{a y^{*}}{h x^{*}+1}}}{a x^{*}},|A|<2\right\},
$$

where

$$
A=\frac{e^{\frac{a y^{*}}{h x^{*}+1}}\left(a h x^{*} y^{*}+\left(h x^{*}+1\right)^{2}\right)}{\lambda\left(h x^{*}+1\right)^{2}}+\frac{a b x^{*} e^{-\frac{a y^{*}}{h x^{*}+1}}}{h x^{*}+1} .
$$

Assuming that $\tilde{b}$ be a small perturbation in $b \equiv b_{1}$, then system (1.2) is described by the following 2-dimensional map:

$$
\left(\begin{array}{c}
U \\
V
\end{array}\right) \rightarrow\left(\begin{array}{c}
\frac{\lambda U \exp \left(-\frac{a V}{1+h U}\right)}{1+\frac{\lambda-1}{k} U \exp \left(-\frac{a V}{1+h U}\right)} \\
\left(b_{1}+\tilde{b}\right) U\left(1-\exp \left(-\frac{a V}{1+h U}\right)\right)
\end{array}\right)
$$

Suppose that $\left(x^{*}, y^{*}\right)$ be unique positive fixed point of the map (5.1), then we consider the translations $u=$ $U-x^{*}$ and $v=V-y^{*}$ for shifting the fixed point of resulting map at origin. Consequently, these translations yield the following map with fixed point at origin:

$$
\left(\begin{array}{l}
u \\
v
\end{array}\right) \rightarrow\left(\begin{array}{ll}
a_{11} & a_{12} \\
a_{21} & a_{22}
\end{array}\right)\left(\begin{array}{l}
u \\
v
\end{array}\right)+\left(\begin{array}{c}
f(u, v) \\
g(u, v)
\end{array}\right)
$$

where

$$
\begin{gathered}
f(u, v)=a_{13} u^{2}+a_{14} u v+a_{15} v^{2}+a_{16} u^{3}+a_{17} u^{2} v+a_{18} u v^{2}+a_{19} v^{3}+O\left((|u|+|v|)^{4}\right), \\
g(u, v)=a_{23} u^{2}+a_{24} u v+a_{25} v^{2}+a_{26} u^{3}+a_{27} u^{2} v+a_{28} u v^{2}+a_{29} v^{3}+O\left((|u|+|v|)^{4}\right), \\
a_{11}=\frac{e^{\frac{a y^{*}}{h x^{*}+1}}\left(a h x^{*} y^{*}+\left(h x^{*}+1\right)^{2}\right)}{\lambda\left(h x^{*}+1\right)^{2}}, a_{12}=-\frac{a x^{*} e^{\frac{a y^{*}}{h x^{*}+1}}}{\lambda\left(h x^{*}+1\right)}, \\
a_{21}=\frac{y^{*}}{x^{*}}-\frac{a\left(b_{1}+\tilde{b}\right) h x^{*} y^{*} e^{-\frac{a y *}{h x^{*}+1}}}{\left(h x^{*}+1\right)^{2}}, a_{22}=\frac{a\left(b_{1}+\tilde{b}\right) x^{*} e^{-\frac{a y^{*}}{h x^{*}+1}}}{h x^{*}+1}, \\
a_{13}=\frac{a h k \lambda y^{*}\left(h x^{*}\left(a y^{*}+2\right)+2\right)-2(\lambda-1)\left(a h x^{*} y^{*}+\left(h x^{*}+1\right)^{2}\right)^{2}}{2 k \lambda\left(h x^{*}+1\right)^{4}} \\
+\frac{(\lambda-1)^{2} x^{*}\left(a h x^{*} y^{*}+\left(h x^{*}+1\right)^{2}\right)^{2}}{k^{2} \lambda^{2}\left(h x^{*}+1\right)^{4}}-\frac{a h(\lambda-1) x^{*} y^{*}\left(h x^{*}\left(a y^{*}+2\right)+2\right)}{2 k \lambda\left(h x^{*}+1\right)^{4}},
\end{gathered}
$$




$$
\begin{gathered}
a_{14}=\frac{a e^{\frac{a y^{*}}{h x^{*}+1}}\left((\lambda-1) x^{*}\left(h x^{*}\left(a y^{*}+2 h x^{*}+3\right)+1\right)-k e^{\frac{a y^{*}}{h x^{*}+1}}\left(h\left(a x^{*} y^{*}+x^{*}\right)+1\right)\right)}{k \lambda^{2}\left(h x^{*}+1\right)^{3}}, \\
a_{15}=\frac{a^{2} x^{*} e^{\frac{a y^{*}}{h x^{*}+1}}\left(k e^{\frac{a y^{*}}{h x^{*}+1}}-\lambda x^{*}+x^{*}\right)}{2 k\left(h \lambda x^{*}+\lambda\right)^{2}}
\end{gathered}
$$$$
\begin{aligned}
a_{17}= & \frac{a h e^{\frac{3 a y^{*}}{h x^{*}+1}}\left(h x^{*}\left(-a^{2} y^{* 2}+2 h x^{*}\left(a y^{*}+1\right)+4\right)-2 a y^{*}+2\right)}{2 \lambda^{4}\left(h x^{*}+1\right)^{5}} \\
& +\frac{2 a(\lambda-1) e^{\frac{2 a y^{*}}{h x^{*}+1}}\left(h x^{*}\left(a y^{*}+2 h x^{*}+3\right)+1\right)\left(h x^{*}\left(a y^{*}+1\right)+1\right)}{k \lambda^{4}\left(h x^{*}+1\right)^{5}} \\
& -\frac{a x^{*}\left(h x^{*}\left(h x^{*}\left(6 h x^{*}\left(a y^{*}+3\right)+a y^{*}\left(a y^{*}+8\right)+6 h^{2} x^{* 2}+20\right)+2\left(a y^{*}+5\right)\right)+2\right)}{2 k^{2} \lambda^{3}\left(h x^{*}+1\right)^{5}(\lambda-1)^{-2} e^{-\frac{a y^{*}}{h x^{*}+1}}},
\end{aligned}
$$$$
a_{16}=3 \Upsilon h^{4} x^{* 2}\left(-a k^{2} y^{*}\left(a y^{*}+2\right) e^{\frac{2 a y^{*}}{h x^{*}+1}}-4 a(\lambda-1) k x^{*} y^{*}\left(a y^{*}+2\right) e^{\frac{a y^{*}}{h x^{*}+1}}\right)
$$$$
+3 \Upsilon h^{4} x^{* 2}\left(3(\lambda-1)^{2} x^{* 2}\left(a y^{*}\left(a y^{*}+6\right)+10\right)\right)
$$$$
+3 \Upsilon h^{2}\left(a k^{2} y^{*}\left(a y^{*}-2\right) e^{\frac{2 a *^{*}}{h x^{*}+1}}-4 a(\lambda-1) k x^{*} y^{*}\left(a y^{*}+4\right) e^{\frac{a y^{*}}{h x^{*}+1}}\right)
$$$$
+3 \Upsilon h^{2}\left((\lambda-1)^{2} x^{* 2}\left(a y^{*}\left(a y^{*}+10\right)+30\right)\right)
$$$$
+\Upsilon h^{3} x^{*}\left(a k^{2} y^{*}\left(a^{2} y^{* 2}-12\right) e^{\frac{2 a y^{*}}{h x^{*}+1}}-4 a(\lambda-1) k x^{*} y^{*}\left(a y^{*}\left(a y^{*}+6\right)+15\right) e^{\frac{a y^{*}}{h x^{*}+1}}\right)
$$$$
+\Upsilon h^{3} x^{*}\left((\lambda-1)^{2} x^{* 2}\left(a y^{*}\left(a y^{*}\left(a y^{*}+12\right)+60\right)+120\right)\right)
$$$$
+\Upsilon\left(18 h^{5}(\lambda-1)^{2} x^{* 5}\left(a y^{*}+2\right)+6 h(\lambda-1)\left((\lambda-1) x^{*}\left(a y^{*}+6\right)-2 a k y^{*} e^{\frac{a y^{*}}{h x^{*}+1}}\right)\right)
$$$$
+\Upsilon\left(6 h^{6}(\lambda-1)^{2} x^{* 6}+6(\lambda-1)^{2}\right), \Upsilon=\frac{e^{\frac{a y^{*}}{h x^{*}+1}}}{6 k^{2} \lambda^{3}\left(h x^{*}+1\right)^{6}},
$$$$
\begin{aligned}
a_{18}= & \frac{a^{2} e^{\frac{2 a y^{*}}{h x^{*}+1}}\left(k e^{\frac{a y^{*}}{h x^{*}+1}}\left(h x^{*}\left(a y^{*}-h x^{*}\right)+1\right)-4(\lambda-1) x^{*}\left(a h x^{*} y^{*}+\left(h x^{*}+1\right)^{2}\right)\right)}{2 k \lambda^{3}\left(h x^{*}+1\right)^{4}} \\
& +\frac{a^{2}(\lambda-1)^{2} x^{* 2} e^{\frac{a y^{*}}{h x^{*}+1}}\left(h x^{*}\left(a y^{*}+3 h x^{*}+4\right)+1\right)}{2 k^{2} \lambda^{3}\left(h x^{*}+1\right)^{4}}
\end{aligned}
$$$$
a_{19}=\frac{a^{3} x^{*} e^{\frac{a y^{*}}{h x^{*}+1}}\left(k^{2} e^{\frac{2 a y^{*}}{h x^{*}+1}}-4(\lambda-1) k x^{*} e^{\frac{a y^{*}}{h x^{*}+1}}+(\lambda-1)^{2} x^{* 2}\right)}{6 k^{2}\left(h \lambda x^{*}+\lambda\right)^{3}},
$$

$$
a_{23}=-\frac{a\left(b_{1}+\tilde{b}\right) h y^{*}\left(h x^{*}\left(a y^{*}+2\right)+2\right)}{2\left(h x^{*}+1\right)^{4} e^{\frac{a y^{*}}{h x^{*}+1}}}, a_{24}=\frac{a\left(b_{1}+\tilde{b}\right) e^{-\frac{a y^{*}}{h x^{*}+1}}\left(h x^{*}\left(a y^{*}+1\right)+1\right)}{\left(h x^{*}+1\right)^{3}},
$$




$$
\begin{gathered}
a_{25}=-\frac{a^{2}\left(b_{1}+\tilde{b}\right) x^{*} e^{-\frac{a y^{*}}{h x^{*}+1}}}{2\left(h x^{*}+1\right)^{2}}, \\
a_{26}=\frac{a\left(b_{1}+\tilde{b}\right) h^{2} y^{*} e^{-\frac{a y^{*}}{h x^{*}+1}}\left(h x^{*}\left(-a^{2} y^{* 2}+3 h x^{*}\left(a y^{*}+2\right)+12\right)-3 a y^{*}+6\right)}{6\left(h x^{*}+1\right)^{6}}, \\
a_{27}=\frac{a\left(b_{1}+\tilde{b}\right) h e^{-\frac{a y^{*}}{h x^{*}+1}}\left(h x^{*}\left(a^{2} y^{* 2}-2 h x^{*}\left(a y^{*}+1\right)-4\right)+2 a y^{*}-2\right)}{2\left(h x^{*}+1\right)^{5}}, \\
a_{28}=\frac{a^{2}\left(b_{1}+\tilde{b}\right) e^{-\frac{a y^{*}}{h x^{*}+1}}\left(h x^{*}\left(h x^{*}-a y^{*}\right)-1\right)}{2\left(h x^{*}+1\right)^{4}}, a_{29}=\frac{a^{3}\left(b_{1}+\tilde{b}\right) x^{*} e^{-\frac{a y^{*}}{h x^{*}+1}}}{6\left(h x^{*}+1\right)^{3}}
\end{gathered}
$$

Now, characteristic polynomial of map (5.2) at its fixed point $(0,0)$ is given by

$$
P(\tau)=\tau^{2}-A(\tilde{b}) \tau+B(\tilde{b})
$$

where

$$
A(\tilde{b}):=\frac{e^{\frac{a y^{*}}{h x^{*}+1}}\left(a h x^{*} y^{*}+\left(h x^{*}+1\right)^{2}\right)}{\lambda\left(h x^{*}+1\right)^{2}}+\frac{a\left(b_{1}+\tilde{b}\right) x^{*} e^{-\frac{a y^{*}}{h x^{*}+1}}}{h x^{*}+1},
$$

and

$$
B(\tilde{b}):=\frac{a\left(y^{*} e^{\frac{a y^{*}}{h x^{*}+1}}+\left(b_{1}+\tilde{b}\right) x^{*}\right)}{h \lambda x^{*}+\lambda} .
$$

Moreover, complex conjugate roots of (5.3) are given by

$$
\tau_{1}=\frac{A(\tilde{b})+\iota \sqrt{4 B(\tilde{b})-A^{2}(\tilde{b})}}{2},
$$

and

$$
\tau_{2}=\frac{A(\tilde{b})-\iota \sqrt{4 B(\tilde{b})-A^{2}(\tilde{b})}}{2} .
$$

Then, simple computation yields that $\left|\tau_{1}\right|=\left|\tau_{2}\right|=\sqrt{B(\tilde{b})}$. Next, in order to verify nondegeneracy conditions, first we see that:

$$
\left(\frac{d\left|\tau_{1,2}\right|}{d \tilde{b}}\right)_{\tilde{b}=0}=\frac{a x^{*}}{2 \lambda\left(h x^{*}+1\right)}>0
$$


Secondly, keeping in view non-resonance, at $\tilde{b}=0$ it is required that $\tau_{1,2}^{n} \neq 1$ for $n=1,2,3,4$, which is equivalent to $A(0) \neq-2,-1,0,2$. Assume that $(a, b, k, h, \lambda) \in C_{N B}$, then it follows that $|A(0)|<2$. Moreover, we have

$$
A(0)=\frac{e^{\frac{a y^{*}}{h x^{*}+1}}\left(a h x^{*} y^{*}+\left(h x^{*}+1\right)^{2}\right)}{\lambda\left(h x^{*}+1\right)^{2}}+\frac{a b_{1} x^{*} e^{-\frac{a y^{*}}{h x^{*}+1}}}{h x^{*}+1}>0 .
$$

Therefore, conditions for non-resonance are automatically satisfied. Furthermore, in order to compute the first Lyapunov exponent, we want to transform Jacobian matrix of (5.2) into canonical form. For this, the following transformation is considered:

$$
\left(\begin{array}{l}
u \\
v
\end{array}\right)=\left(\begin{array}{cc}
a_{12} & 0 \\
\alpha-a_{11} & -\beta
\end{array}\right)\left(\begin{array}{c}
w \\
z
\end{array}\right)
$$

where

$$
\alpha:=\frac{A(0)}{2} \text { and } \beta:=\frac{\sqrt{4 B(0)-A^{2}(0)}}{2} .
$$

Consequently, from (5.2) and (5.4), it follows that

$$
\left(\begin{array}{c}
w \\
z
\end{array}\right) \rightarrow\left(\begin{array}{cc}
\alpha & -\beta \\
\beta & \alpha
\end{array}\right)\left(\begin{array}{c}
w \\
z
\end{array}\right)+\left(\begin{array}{c}
M(w, z) \\
N(w, z)
\end{array}\right)
$$

where

$$
M(w, z):=\frac{1}{a_{12}} f\left(a_{12} w,\left(\alpha-a_{11}\right) w-\beta z\right)
$$

and

$$
N(w, z):=-\frac{a_{11}-\alpha}{a_{12} \beta} f\left(a_{12} w,\left(\alpha-a_{11}\right) w-\beta z\right)-\frac{1}{\beta} g\left(a_{12} w,\left(\alpha-a_{11}\right) w-\beta z\right) .
$$

Keeping in view the normal forms theory related to bifurcation analysis $[19,28,43,53,57]$, at $(w, z, \tilde{b})=(0,0,0)$ the first Lyapunov exponent is computed as follows:

$$
L=-\operatorname{Re}\left(\frac{\left(1-2 \tau_{1}\right) \tau_{2}^{2}}{1-\tau_{1}} \tau_{20} \tau_{11}\right)-\frac{1}{2}\left|\tau_{11}\right|^{2}-\left|\tau_{02}\right|^{2}+\operatorname{Re}\left(\tau_{2} \tau_{21}\right)
$$

where

$$
\begin{gathered}
\tau_{20}=\frac{1}{8}\left[M_{w w}-M_{z z}+2 N_{w z}+i\left(N_{w w}-N_{z z}-2 M_{w z}\right)\right], \\
\tau_{11}=\frac{1}{4}\left[M_{w w}+M_{z z}+i\left(N_{w w}+N_{z z}\right)\right], \\
\tau_{02}=\frac{1}{8}\left[M_{w w}-M_{z z}-2 N_{w z}+i\left(N_{w w}-N_{z z}+2 M_{w z}\right)\right],
\end{gathered}
$$


and

$$
\tau_{21}=\frac{1}{16}\left[M_{w w w}+M_{w z z}+N_{w w z}+N_{z z z}+i\left(N_{w w w}+N_{w z z}-M_{w w z}-M_{z z z}\right)\right] .
$$

Then, due to above calculation, one has the following Theorem:

Theorem 5.1. Suppose that $L \neq 0$, then unique positive equilibrium point $\left(x^{*}, y^{*}\right)$ of system (1.2) undergoes Neimark-Sacker bifurcation when the bifurcation parameter $b$ varies in a small neighborhood of $b_{1}=$ $\frac{h \lambda x^{*}+\lambda-a y^{*} e^{\frac{a y^{*}}{h x^{*}+1}}}{a x^{*}}$. Moreover, if $L>0$, then a stable invariant closed curve bifurcates from the equilibrium point for $b>b_{1}$, and if $L<0$, then an unstable invariant closed curve bifurcates from the equilibrium point for $b<b_{1}$.

\section{Chaos control}

Control of chaos and bifurcation is a theme of remarkable interest. In particular, bifurcation and chaos control methods are more applicable for models of discrete nature because these system are of complex behavior as compare to continuous ones. On the other hand, chaos control methods are widely used in almost all branches of applied science and engineering.

This section is dedicated for implementation of chaos control methods to system (1.2). For this, three chaos control methods are implemented to system (1.2). Implementation of chaos control methods for discrete-time systems is topic of great interest. Recently, many dynamical systems have been discussed with implementation of strategies related to chaos and bifurcations control $[1,11,12,40,42,44,54,60,61]$. In [15], variety of chaos control methods have been implemented to a discrete class of predator-prey interaction. A generalized hybrid control method based on parameter perturbation and state feedback control has been proposed in [16]. A new chaos control strategy of exponential type has been introduced in [17] for controlling fluctuating and chaotic behavior of discrete-time models. We apply two frequently used and one recently developed chaos control methods to system (1.2) as follows.

First, we consider Ott-Grebogi-Yorke (OGY) method (see [41]). An application of OGY feedback control method to system (1.2) yields the following control system:

$$
\begin{aligned}
x_{n+1} & =\frac{\lambda x_{n} \exp \left(-\frac{a y_{n}}{1+h x_{n}}\right)}{1+\frac{\lambda-1}{k} x_{n} \exp \left(-\frac{a y_{n}}{1+h x_{n}}\right)}, \\
y_{n+1} & =\left(b-k_{1}\left(x_{n}-x^{*}\right)-k_{2}\left(y_{n}-y^{*}\right)\right) x_{n}\left(1-\exp \left(-\frac{a y_{n}}{1+h x_{n}}\right)\right),
\end{aligned}
$$

where $k_{1}$ and $k_{2}$ are control parameters, and $\left(x^{*}, y^{*}\right)$ is interior equilibrium point of system (1.2). In order to see the controllability of system (6.1), the Jacobian matrix for this controlled system at $\left(x^{*}, y^{*}\right)$ is computed as follows:

$$
J_{C}\left(x^{*}, y^{*}\right)=\left(\begin{array}{cc}
\frac{e^{\frac{a y^{*}}{h x^{*}+1}}\left(a h x^{*} y^{*}+\left(h x^{*}+1\right)^{2}\right)}{\lambda\left(h x^{*}+1\right)^{2}} & -\frac{a x^{*} e^{\frac{a y^{*}}{h x^{*}+1}}}{\lambda\left(h x^{*}+1\right)} \\
\frac{y^{*}}{x^{*}}-\frac{k_{1} y^{*}}{b}-\frac{a b h x^{*} y^{*} e^{-\frac{a y *}{h x^{*}+1}}}{\left(h x^{*}+1\right)^{2}} & \frac{a b x^{*} e^{-\frac{a y^{*}}{h x^{*}+1}}}{h x^{*}+1}-\frac{k_{2} y^{*}}{b}
\end{array}\right) .
$$


In addition, the characteristic polynomial for $J_{C}\left(x^{*}, y^{*}\right)$ is given by:

$$
\begin{aligned}
F(\eta)= & \eta^{2}-\left(\frac{e^{\frac{a y^{*}}{h x^{*}+1}}\left(a h x^{*} y^{*}+\left(h x^{*}+1\right)^{2}\right)}{\lambda\left(h x^{*}+1\right)^{2}}+\frac{a b x^{*} e^{-\frac{a y^{*}}{h x^{*}+1}}}{h x^{*}+1}-\frac{k_{2} y^{*}}{b}\right) \eta \\
& +\frac{a b^{2} x^{*}\left(h x^{*}+1\right)-y e^{\frac{a y^{*}}{h x^{*}+1}}\left(\left(h x^{*}+1\right)\left(-a b+a k_{1} x^{*}+h k_{2} x^{*}+k_{2}\right)+a h k_{2} x^{*} y^{*}\right)}{b \lambda\left(h x^{*}+1\right)^{2}} .
\end{aligned}
$$

Taking into account the controllability of system (6.1), the following Lemma is presented.

Lemma 6.1. System (6.1) is controllable if the following condition is satisfied:

$$
\begin{aligned}
& \left|\frac{e^{\frac{a y^{*}}{h x^{*}+1}}\left(a h x^{*} y^{*}+\left(h x^{*}+1\right)^{2}\right)}{\lambda\left(h x^{*}+1\right)^{2}}+\frac{a b x^{*} e^{-\frac{a y^{*}}{h x^{*}+1}}}{h x^{*}+1}-\frac{k_{2} y^{*}}{b}\right| \\
& <1+\frac{a b^{2} x^{*}\left(h x^{*}+1\right)-y e^{\frac{a y^{*}}{h x^{*}+1}}\left(\left(h x^{*}+1\right)\left(-a b+a k_{1} x^{*}+h k_{2} x^{*}+k_{2}\right)+a h k_{2} x^{*} y^{*}\right)}{b \lambda\left(h x^{*}+1\right)^{2}}<2 .
\end{aligned}
$$

Secondly, we apply hybrid control method [35] to system (1.2) to get the following control system:

$$
\begin{aligned}
& x_{n+1}=\alpha\left(\frac{\lambda x_{n} \exp \left(-\frac{a y_{n}}{1+h x_{n}}\right)}{1+\frac{\lambda-1}{k} x_{n} \exp \left(-\frac{a y_{n}}{1+h x_{n}}\right)}\right)+(1-\alpha) x_{n}, \\
& y_{n+1}=\alpha\left(b x_{n}\left(1-\exp \left(-\frac{a y_{n}}{1+h x_{n}}\right)\right)\right)+(1-\alpha) y_{n},
\end{aligned}
$$

where $0<\alpha<1$ is control parameter for hybrid control method, which is based on parameter perturbation and state feedback control. Again controllability of system (6.2) depends upon stability of this system at its positive fixed point. To see this stability behavior, the Jacobian matrix $J_{H}\left(x^{*}, y^{*}\right)$ of system (6.2) at its unique positive fixed point $\left(x^{*}, y^{*}\right)$ is given as follows:

$$
J_{H}\left(x^{*}, y^{*}\right)=\left(\begin{array}{cc}
\frac{\alpha e^{\frac{a y^{*}}{h x^{*}+1}}\left(a h x^{*} y^{*}+\left(h x^{*}+1\right)^{2}\right)}{\lambda\left(h x^{*}+1\right)^{2}}+1-\alpha & -\frac{\alpha a x^{*} e^{\frac{a y^{*}}{h x^{*}+1}}}{\lambda\left(h x^{*}+1\right)} \\
\frac{\alpha y^{*}}{x^{*}}-\frac{\alpha a b h x^{*} y^{*} e^{-\frac{a y^{*}}{h x^{*}+1}}}{\left(h x^{*}+1\right)^{2}} & \frac{\alpha a b x^{*} e^{-\frac{a y^{*}}{h x^{*}+1}}}{h x^{*}+1}+1-\alpha
\end{array}\right) .
$$

In addition, the characteristic polynomial for $J_{H}\left(x^{*}, y^{*}\right)$ is computed as follows:

$$
\begin{aligned}
F(\mu)= & \mu^{2}-\left(2-2 \alpha+\frac{\alpha e^{\frac{a y^{*}}{h x^{*}+1}}\left(a h x^{*} y^{*}+\left(h x^{*}+1\right)^{2}\right)}{\lambda\left(h x^{*}+1\right)^{2}}+\frac{\alpha a b x^{*} e^{-\frac{a y^{*}}{h x^{*}+1}}}{h x^{*}+1}\right) \mu \\
& +\frac{e^{-\frac{a y^{*}}{h x^{*}+1}}\left(e^{\frac{a y^{*}}{h x^{*}+1}}\left(a \alpha^{2} b x^{*}+(\alpha-1)^{2} \lambda\left(h x^{*}+1\right)\right)-a(\alpha-1) \alpha b \lambda x^{*}\right)}{h \lambda x^{*}+\lambda} \\
& -\frac{\alpha e^{\frac{a y^{*}}{h x^{*}+1}}\left(\alpha\left(h x^{*}\left(h x^{*}+2\right)-a y\right)-h x^{*}\left(a y^{*}+h x^{*}+2\right)+\alpha-1\right)}{\lambda\left(h x^{*}+1\right)^{2}} .
\end{aligned}
$$

Taking into account the controllability of system (6.2), the following Lemma is presented. 
Lemma 6.2. Positive fixed point $\left(x^{*}, y^{*}\right)$ of system (6.2) is a sink if the following condition is satisfied:

$$
\begin{aligned}
& \left|2-2 \alpha+\frac{\alpha e^{\frac{a y^{*}}{h x^{*}+1}}\left(a h x^{*} y^{*}+\left(h x^{*}+1\right)^{2}\right)}{\lambda\left(h x^{*}+1\right)^{2}}+\frac{\alpha a b x^{*} e^{-\frac{a y^{*}}{h x^{*}+1}}}{h x^{*}+1}\right| \\
& <1+\frac{e^{-\frac{a y^{*}}{h x^{*}+1}}\left(e^{\frac{a y^{*}}{h x^{*}+1}}\left(a \alpha^{2} b x^{*}+(\alpha-1)^{2} \lambda\left(h x^{*}+1\right)\right)-a(\alpha-1) \alpha b \lambda x^{*}\right)}{h \lambda x^{*}+\lambda} \\
& -\frac{\alpha e^{\frac{a y^{*}}{h x^{*}+1}}\left(\alpha\left(h x^{*}\left(h x^{*}+2\right)-a y\right)-h x^{*}\left(a y^{*}+h x^{*}+2\right)+\alpha-1\right)}{\lambda\left(h x^{*}+1\right)^{2}}<2 .
\end{aligned}
$$

Thirdly, our aim is to apply recently proposed exponential chaos control technique to system (1.2) [17]. With application of exponential type chaos control strategy, we obtain the following control system:

$$
\begin{aligned}
& x_{n+1}=\exp \left(-s_{1}\left(x_{n}-x^{*}\right)\right)\left(\frac{\lambda x_{n} \exp \left(-\frac{a y_{n}}{1+h x_{n}}\right)}{1+\frac{\lambda-1}{k} x_{n} \exp \left(-\frac{a y_{n}}{1+h x_{n}}\right)}\right), \\
& y_{n+1}=\exp \left(-s_{2}\left(y_{n}-y^{*}\right)\right)\left(b x_{n}\left(1-\exp \left(-\frac{a y_{n}}{1+h x_{n}}\right)\right)\right),
\end{aligned}
$$

where $s_{1}$ and $s_{2}$ are control parameters for exponential control strategy and $\left(x^{*}, y^{*}\right)$ be interior (positive) fixed point of system (1.2). Next, controllability of system (6.3) is directly related to stability of system about its positive fixed point $\left(x^{*}, y^{*}\right)$. For this, Jacobian matrix $J_{E}\left(x^{*}, y^{*}\right)$ of system $(6.3)$ about $\left(x^{*}, y^{*}\right)$ is computed as follows:

$$
J_{E}\left(x^{*}, y^{*}\right)=\left(\begin{array}{cc}
\frac{\left(\left(h x^{*}+1\right)^{2}+a h x^{*} y^{*}\right)\left(\lambda-\frac{x^{*}(\lambda-1)}{k}\right)}{\left(h x^{*}+1\right)^{2} \lambda}-x^{*} s_{1} & -\frac{a x^{*}\left(x^{*}-\lambda x^{*}+k \lambda\right)}{k\left(h x^{*}+1\right) \lambda} \\
b\left(1-\frac{k\left(\frac{a h x^{*} y^{*}}{\left(h x^{*}+1\right)^{2}}+1\right)}{x^{*}-\lambda x^{*}+k \lambda}\right) & b x^{*}\left(\frac{k\left(a+\left(h x^{*}+1\right) s_{2}\right)}{\left(h x^{*}+1\right)\left(x^{*}-\lambda x^{*}+k \lambda\right)}-s_{2}\right)
\end{array}\right) .
$$

Moreover, the characteristic polynomial for $J_{E}\left(x^{*}, y^{*}\right)$ is computed as follows:

$$
\begin{aligned}
F(\varphi)= & \varphi^{2}-\left(b x^{*}\left(\frac{k\left(a+s_{2}\left(h x^{*}+1\right)\right)}{\left(h x^{*}+1\right)\left(k \lambda-\lambda x^{*}+x^{*}\right)}-s_{2}\right)+\frac{\left(\frac{x^{*}-\lambda x^{*}}{k}+\lambda\right)\left(a h x^{*} y^{*}+\left(h x^{*}+1\right)^{2}\right)}{\lambda\left(h x^{*}+1\right)^{2}}-s_{1} x^{*}\right) \varphi \\
& +\left(\frac{\left.\left(h x^{*}+1\right)^{2}+a h x^{*} y^{*}\right)\left(\lambda-\frac{x^{*}(\lambda-1)}{k}\right)}{\left(h x^{*}+1\right)^{2} \lambda}-x^{*} s_{1}\right)\left(b x^{*}\left(\frac{k\left(a+\left(h x^{*}+1\right) s_{2}\right)}{\left(h x^{*}+1\right)\left(x^{*}-\lambda x^{*}+k \lambda\right)}-s_{2}\right)\right) \\
& +\left(b\left(1-\frac{k\left(\frac{a h x^{*} y^{*}}{\left(h x^{*}+1\right)^{2}}+1\right)}{x^{*}-\lambda x^{*}+k \lambda}\right)\right)\left(\frac{a x^{*}\left(x^{*}-\lambda x^{*}+k \lambda\right)}{k\left(h x^{*}+1\right) \lambda}\right) .
\end{aligned}
$$

Taking into account the controllability of system (6.3), the following Lemma is presented. 


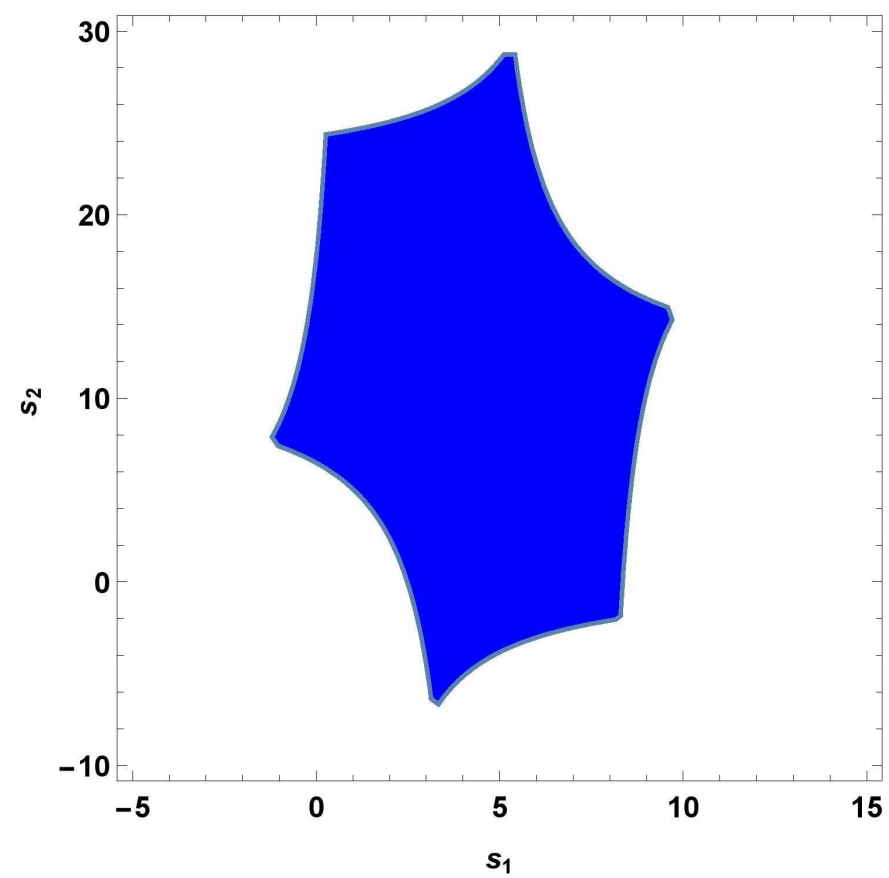

Figure 5. Stability region for system (6.3) with $a=11.5, b=0.68, h=0.9, \lambda=1.9$ and $k=3$.

Lemma 6.3. Positive fixed point $\left(x^{*}, y^{*}\right)$ of system (6.3) is a sink if the following condition is satisfied:

$$
\begin{aligned}
& \left|b x^{*}\left(\frac{k\left(a+s_{2}\left(h x^{*}+1\right)\right)}{\left(h x^{*}+1\right)\left(k \lambda-\lambda x^{*}+x^{*}\right)}-s_{2}\right)+\frac{\left(\frac{x^{*}-\lambda x^{*}}{k}+\lambda\right)\left(a h x^{*} y^{*}+\left(h x^{*}+1\right)^{2}\right)}{\lambda\left(h x^{*}+1\right)^{2}}-s_{1} x^{*}\right| \\
& <1+\left(\frac{\left(\left(h x^{*}+1\right)^{2}+a h x^{*} y^{*}\right)\left(\lambda-\frac{x^{*}(\lambda-1)}{k}\right)}{\left(h x^{*}+1\right)^{2} \lambda}-x^{*} s_{1}\right)\left(b x^{*}\left(\frac{k\left(a+\left(h x^{*}+1\right) s_{2}\right)}{\left(h x^{*}+1\right)\left(x^{*}-\lambda x^{*}+k \lambda\right)}-s_{2}\right)\right) \\
& \quad+\left(b\left(1-\frac{k\left(\frac{a h x^{*} y^{*}}{\left(h x^{*}+1\right)^{2}}+1\right)}{x^{*}-\lambda x^{*}+k \lambda}\right)\right)\left(\frac{a x^{*}\left(x^{*}-\lambda x^{*}+k \lambda\right)}{k\left(h x^{*}+1\right) \lambda}\right)<2 .
\end{aligned}
$$

Note that variations of parametric values affect control performance of system (6.3). For example, if we choose $a=11.5, b=0.68, h=0.9, \lambda=1.9$ and $k=3$, then controllability (stability) region (blue region) of system (6.3) is depicted in Figure 5.

\section{NumericAl SIMUlation AND DisCUSSION}

For the verification of theoretical discussion, we assume that $(\lambda, a, h, k)=(10.4,0.5,5.8,1.2), b \in[1,45]$ and $\left(x_{0}, y_{0}\right)=(0.67,16)$. Then, system $(1.2)$ undergoes transcritical bifurcation around its boundary equilibrium $(k, 0)=(1.2,0)$ when $b$ passes through the critical value $b_{0}=13.2667$. On the other hand, system (1.2) undergoes Neimark-Sacker bifurcation around its interior equilibrium $\left(x^{*}, y^{*}\right)=(0.673646,16.0342)$ when $b$ passes through the critical value $b_{1}=29.575$. At $(\lambda, a, h, k, b)=(10.4,0.5,5.8,1.2,29.575)$, the multipliers of system $(1.2)$ are $\mu_{1}=0.764824+0.644235 i$ and $\mu_{2}=0.764824-0.644235 i$ with $\left|\mu_{1}\right|=\left|\mu_{2}\right|=1$. Moreover, bifurcation diagrams and maximum Lyapunov exponents (MLE) of system are depicted in Figure 6. Moreover, for $b \in[36,40]$ local 


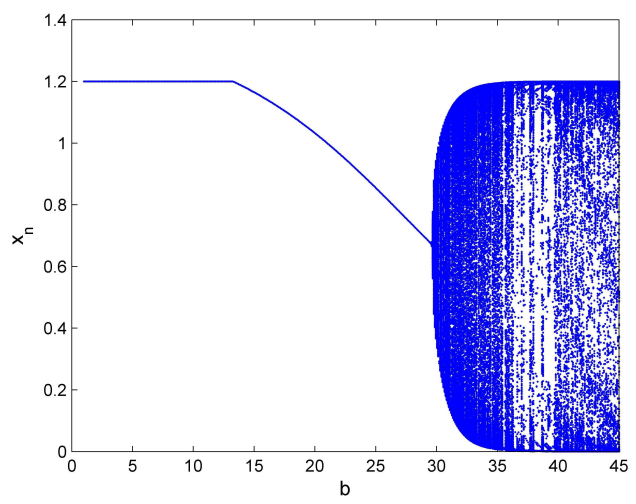

(a) Bifurcation diagram for $x_{n}$

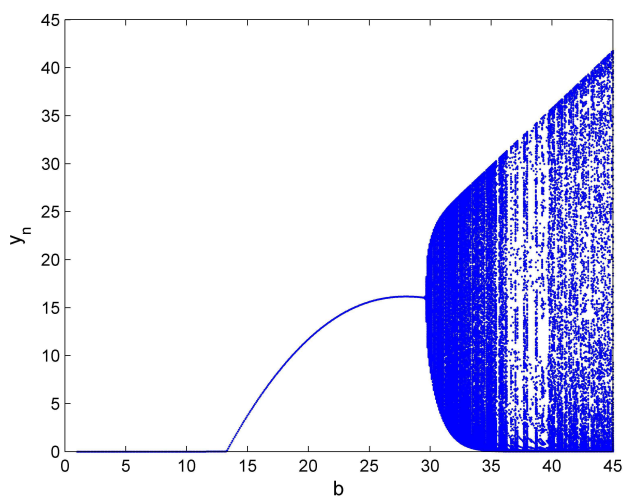

(b) Bifurcation diagram for $y_{n}$

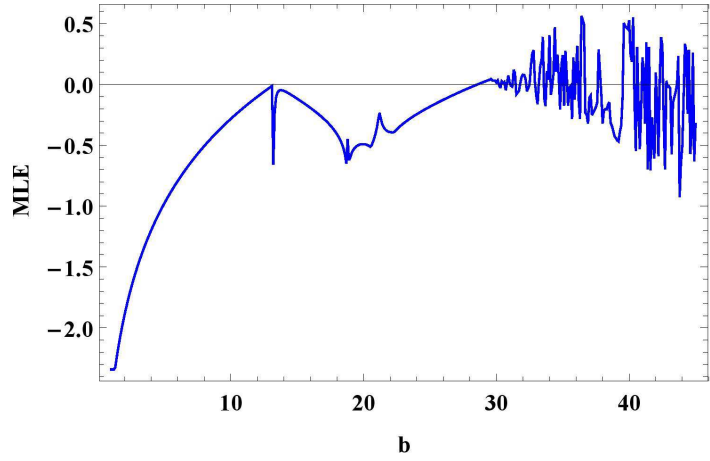

(c) Maximum Lyapunov exponents

FiguRE 6. Bifurcation diagrams and MLE for system $(1.2)$ with $(\lambda, a, h, k)=(10.4,0.5,5.8,1.2)$, $b \in[1,45]$ and $\left(x_{0}, y_{0}\right)=(0.67,16)$.

amplification of Figure 6 is depicted in Figure 7. On the other hand, for $b=29.5,29.575,29.58,30$ phase portraits of system (1.2) are depicted in Figure 8.

Next, we check the validity of chaos control strategies discussed in Section 6. For this, we choose $(\lambda, a, h, k, b)=(10.4,0.5,5.8,1.2,40)$. At these parametric values system (1.2) has unique positive fixed point $\left(x^{*}, y^{*}\right)=(0.360595,12.5197)$. Clearly, this fixed point is source because multipliers of system (1.2) are $\mu_{1}=1.01709+0.815335 i$ and $\mu_{2}=1.01709-0.815335 i$ with $\left|\mu_{1}\right|=\left|\mu_{2}\right|=1.30355>1$. First, we apply OGY method for these parametric values. In order to see the controllability of system (6.1), the Jacobian matrix for this controlled system at $(0.360595,12.5197)$ reduces to:

$$
J_{C}(0.360595,12.5197)=\left(\begin{array}{cc}
1.72623 & -0.0424813 \\
27.4862-0.312993 k_{1} & 0.307956-0.312993 k_{2}
\end{array}\right) .
$$

The characteristic polynomial for $J_{C}(0.360595,12.5197)$ is given by

$$
P(\mu)=\mu^{2}+\left(0.312993 k_{2}-2.03419\right) \mu+1.69925-0.0132964 k_{1}-0.540299 k_{2} .
$$

An application of Jury condition gives that system (6.1) is controllable if $\left|0.312993 k_{2}-2.03419\right|<2.69925-$ $0.0132964 k_{1}-0.540299 k_{2}<2$. Furthermore, the controllable region in $k_{1} k_{2}$-plane with OGY method is shown in Figure 9. It must be noticed that the stability region depicted in Figure 9 is a region bounded by the following 


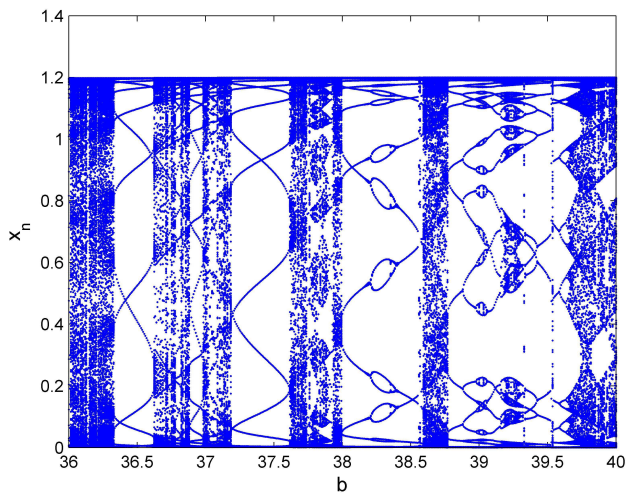

(a) Local amplification of Fig. 11a

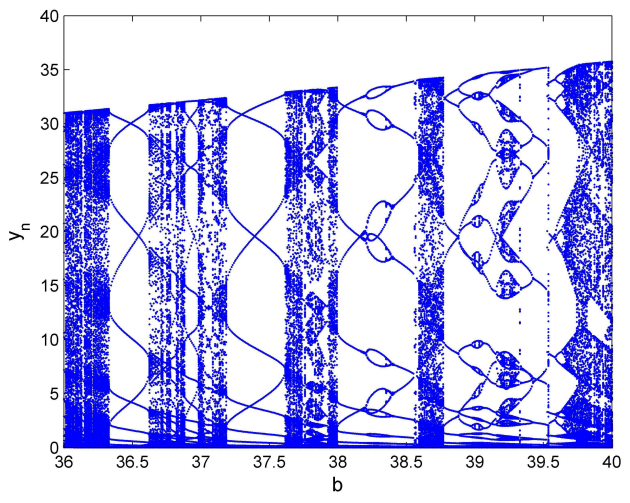

(b) Local amplification of Fig. 11a

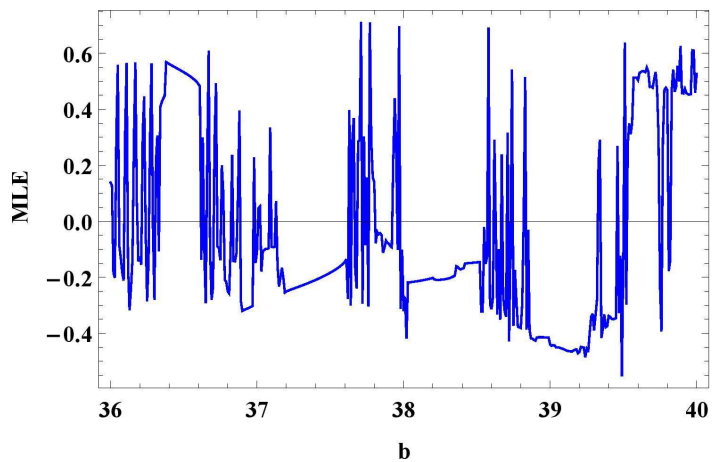

(c) Local amplification of Fig. 11c

Figure 7. Local amplification of Figure 6 with $(\lambda, a, h, k)=(10.4,0.5,5.8,1.2), b \in[36,40]$ and $\left(x_{0}, y_{0}\right)=(0.67,16)$.

three lines of marginal stability:

$$
\begin{aligned}
& L_{1}: k_{2}=2.92584-0.0584956 k_{1}, \\
& L_{2}: k_{2}=5.54727-0.0155825 k_{1},
\end{aligned}
$$

and

$$
L_{3}: k_{2}=1.29419-0.0246093 k_{1} .
$$

It is worthwhile to mention that the marginal stability lines $L_{1}, L_{2}$ and $L_{3}$ are consequences of $P(1)=0$, $P(-1)=0$ and $P(0)=1$, respectively.

Secondly, we see the effectiveness of hybrid control method. For this, similar parametric values are chosen, that is, $(\lambda, a, h, k, b)=(10.4,0.5,5.8,1.2,40)$ are used in system $(6.2)$. Then, simple calculation yields that system (6.2) is controllable if $-0.0514052<\alpha<0$, which is impossible because $0<\alpha<1$. Therefore, we are unable to control system (1.2) at these parametric values with hybrid control method. The failure of hybrid control method is due to the choice of bifurcation parameter $b=40$, which located at most right end of chaotic region $[29.575,45]$. On the other hand, if we choose $b=35$, then system (6.2) is controllable if $0<\alpha<0.257381$. Thus 


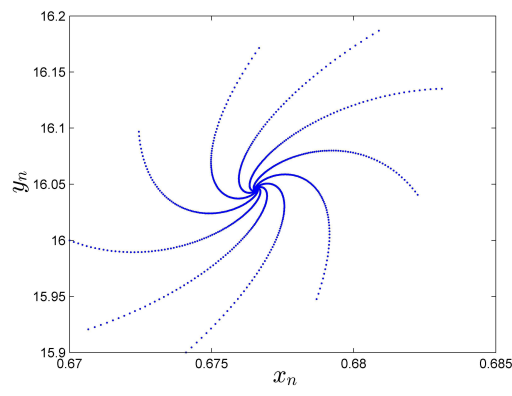

(a) Phase portrait for $b=29.5$

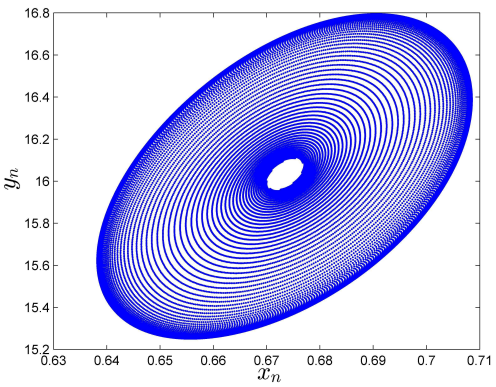

(c) Phase portrait for $b=29.58$

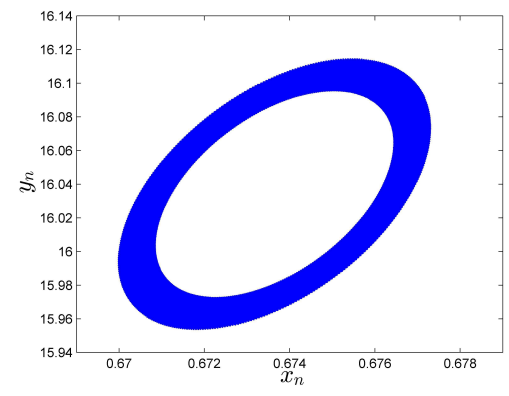

(b) Phase portrait for $b=29.575$

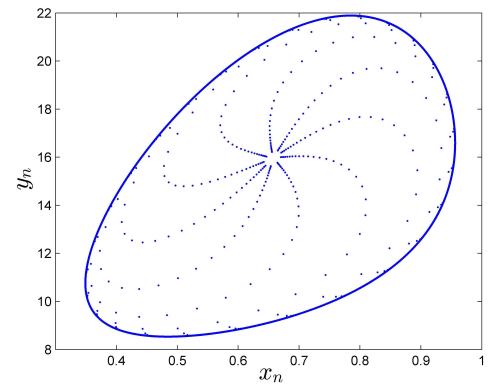

(d) Phase portrait for $b=30$

Figure 8. Phase portraits of system $(1.2)$ for $(\lambda, a, h, k)=(10.4,0.5,5.8,1.2),\left(x_{0}, y_{0}\right)=$ $(0.67,16)$ and with different values of $b$.

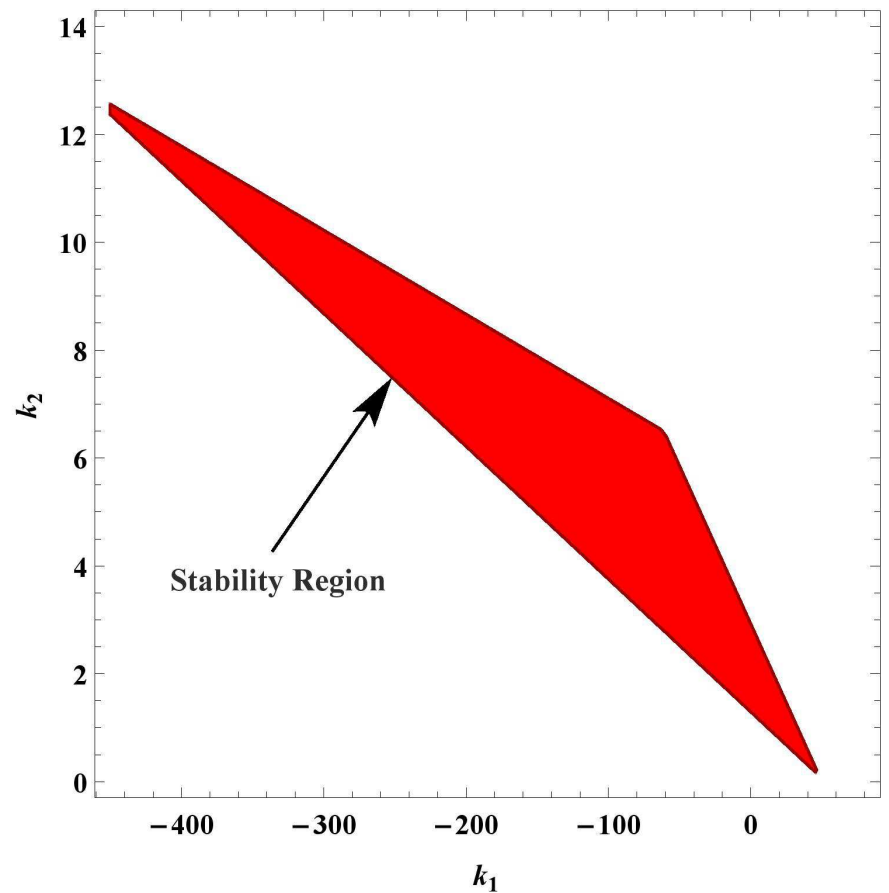

Figure 9. Stability region for system (6.1) with $(\lambda, a, h, k, b)=(10.4,0.5,5.8,1.2,40)$. 
TABLE 2. Controllable intervals for system (6.2) with various values of $b$ in chaotic region.

\begin{tabular}{cc}
\hline Value of bifurcation parameter & Stability interval of system $(6.2)$ \\
\hline$b=38$ & $0<\alpha<0.0357404$ \\
$b=37$ & $0<\alpha<0.0960227$ \\
$b=36$ & $0<\alpha<0.16947$ \\
$b=35$ & $0<\alpha<0.257381$ \\
$b=34$ & $0<\alpha<0.36072$ \\
$b=33$ & $0<\alpha<0.479921$ \\
$b=32$ & $0<\alpha<0.614775$ \\
$b=31$ & $0<\alpha<0.764383$ \\
$b=30$ & $0<\alpha<0.927241$ \\
\hline
\end{tabular}

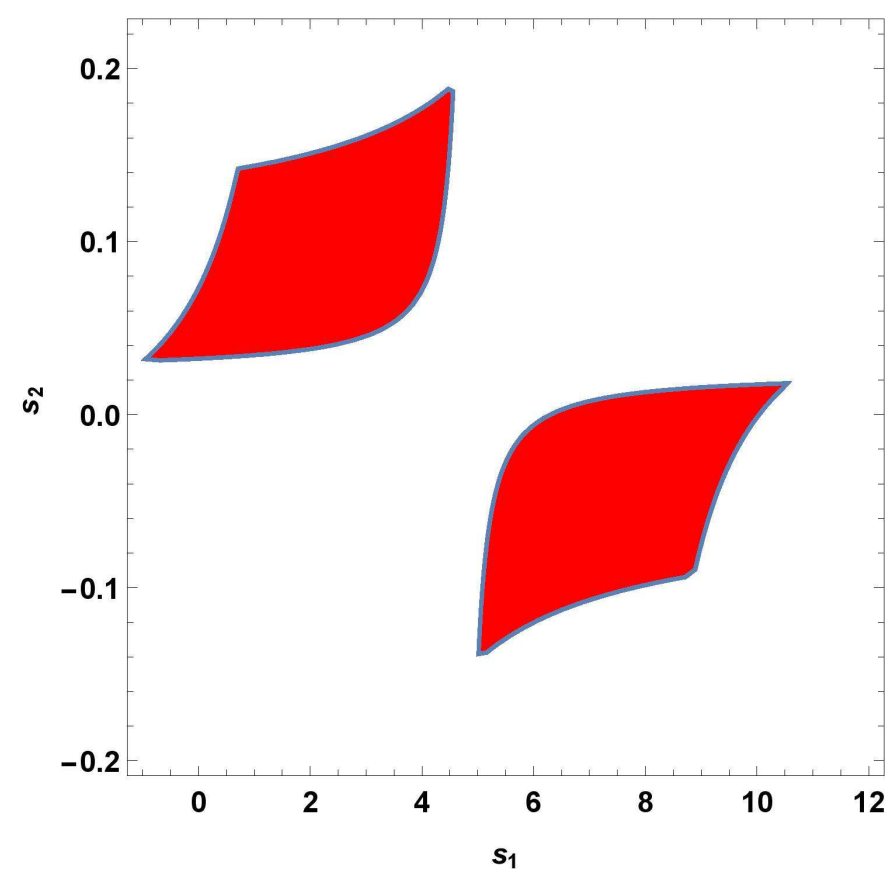

Figure 10. Stability regions for system $(6.3)$ with $(\lambda, a, h, k, b)=(10.4,0.5,5.8,1.2,40)$.

length of controllability interval can be increased by shifting the value of bifurcation parameter at the left end of chaotic region ( $c f$. Tab. 2).

Next, we check exponential-type chaos control method by taking $\lambda=10.4, a=0.5, h=5.8, k=1.2$ and $b=40$ in control system (6.3). In order to see the controllability of system (6.3), the Jacobian matrix for this controlled system at $(0.360595,12.5197)$ reduces to:

$$
J_{E}(0.360595,12.5197)=\left(\begin{array}{cc}
1.72623-0.360595 s_{1} & -0.0424811 \\
27.4862 & 0.307953-12.5197 s_{2}
\end{array}\right) .
$$

The characteristic polynomial for $J_{E}(0.360595,12.5197)$ is given by

$$
P(\varphi)=\varphi^{2}+\left(0.361 s_{1}+12.52 s_{2}-2.034\right) \varphi+1.699-21.6 s_{2}+s_{1}\left(4.5 s_{2}-0.11\right) .
$$




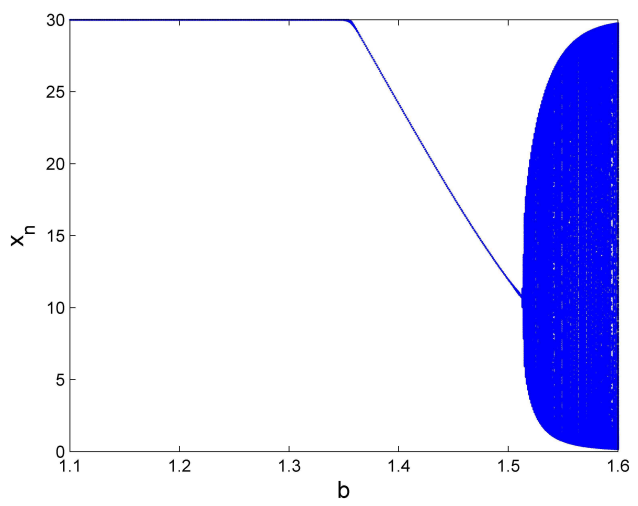

(a) Bifurcation diagram for $x_{n}$

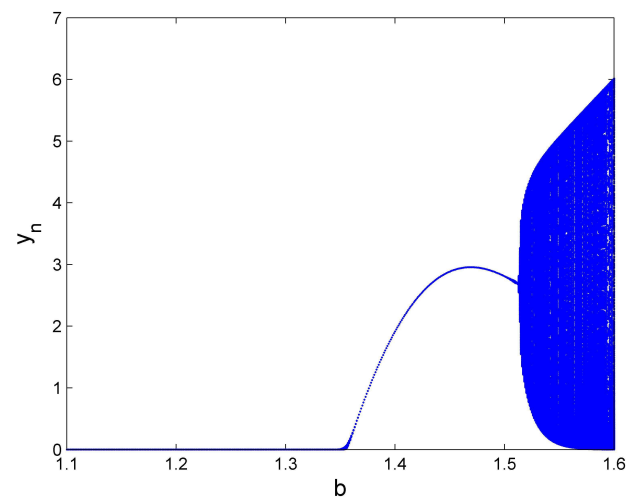

(b) Bifurcation diagram for $y_{n}$

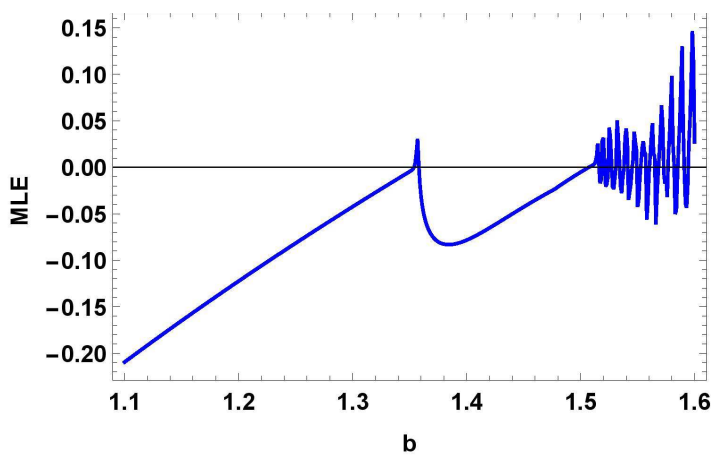

(c) Maximum Lyapunov exponents

FiguRE 11. Bifurcation diagrams and MLE for system (1.2) with $k=30, \lambda=1.309, a=2.207$, $h=2.96, b \in[1.1,1.6]$ and $\left(x_{0}, y_{0}\right)=(10.8,2.696)$.

An application of Jury condition gives that system (6.3) is controllable if $\left|0.361 s_{1}+12.52 s_{2}-2.034\right|<$ $2.699-21.6 s_{2}+s_{1}\left(4.5 s_{2}-0.11\right)<2$. Moreover, the controllable region in $s_{1} s_{2}$-plane with exponential-type method is shown in Figure 10. It is easy to see that in Figure 10 two red regions are depicted as stability (controllable) regions for system (6.3). Consequently, at $\lambda=10.4, a=0.5, h=5.8, k=1.2$ and $b=40$, OGY and exponential-type control methods are partially successful for controlling fluctuating and bifurcating behavior due to appearance of Neimark-Sacker bifurcation. On the other hand, hybrid control method is unable to avoid the population densities from unpredictable situation at extreme right part of chaotic region.

\section{CONCLUding REMARKS}

This paper is concerned to investigation of some qualitative aspects of a discrete-time prey-predator model. The model is a modification of classical Nicholson-Bailey framework related to host-parasitoid interaction with implementation of Beverton-Holt growth function for prey population and type-II functional response. Persistence of solutions is discussed with implementation of method of comparison. Moreover, the existence of fixed points along their stability analysis are carried out. It is proved that system undergoes transcritical bifurcation around its boundary fixed point. On the other hand, discrete prey-predator model undergoes Neimark-Sacker bifurcation around its interior fixed point. Three chaos control methods are applied for control of bifurcating and chaotic behavior of system. Our investigation reveals that OGY and exponential-type control methods are more effective as compare to hybrid control strategy for stability of corresponding controlled systems. Furthermore, in order to validate our theoretical findings with respect to observed field data related to predator-prey interaction, 
TABle 3. Parameter estimates of system (1.2) from observed data.

\begin{tabular}{ccc}
\hline Parameter of system (1.2) & Parameter estimate & Reference \\
\hline$k$ & 30 & {$[29]$} \\
$\lambda$ & 1.309 & {$[49]$} \\
$a$ & 2.207 & {$[45]$} \\
$h$ & 2.96 & {$[47]$} \\
$b$ & 0.046 & {$[14]$} \\
\hline
\end{tabular}

the following parametric estimations are presented: Taking into account the estimated parametric values from observed field data in Table 3, we have $\frac{a b k}{1+h k}=0.033916<1$. Consequently, boundary fixed point $(k, 0)$ is a sink for these parametric values. Furthermore, if we vary bifurcation parameter $b$ and keep all other parametric values fixed (that is, $k=30, \lambda=1.309, a=2.207$ and $h=2.96$ ), then boundary equilibrium is a sink if $0<b<1.35629$, and it undergoes transcritical bifurcation at $b \approx 1.35629$. On the other hand, positive equilibrium point is a sink if $1.35629<b<1.51164$. At $b \approx 1.51164$, the positive fixed point $\left(x^{*}, y^{*}\right)=(10.8055,2.69622)$ undergoes Neimark-Sacker bifurcation. This whole scenario is depicted in Figure 11. Consequently, conversion efficiency from prey to predator plays vital role for altering the dynamics of predator-prey interaction. Arguing as in [13], the conversion efficiency may increase due to change in temperature (warming) and in a result simulated population dynamics reveal a destabilisation of predator-prey interaction with warming. On the other hand, conversion efficiency must be high when the actual prey biomass is low and must be low when actual prey biomass is high. Furthermore, the impact of changes in edibility and the conversion efficiency on the effective prey biomass must outweigh the impact of changes in actual prey biomass [52].

\section{Appendix A. Proof of Theorem 2.1}

Suppose that $x_{0}>0$ and $y_{0}>0$, then every solution $\left\{\left(x_{n}, y_{n}\right)\right\}$ of the system (1.2) satisfies $x_{n}>0$ and $y_{n}>0$ for all $n \geq 0$. Taking into account the positivity of solutions of system (1.2) and considering the first equation of the system (1.2) we have

$$
\begin{aligned}
x_{n+1} & =\frac{\lambda x_{n} \exp \left(-\frac{a y_{n}}{1+h x_{n}}\right)}{1+\frac{\lambda-1}{k} x_{n} \exp \left(-\frac{a y_{n}}{1+h x_{n}}\right)} \\
& =\frac{\lambda x_{n}}{\exp \left(\frac{a y_{n}}{1+h x_{n}}\right)+\frac{\lambda-1}{k} x_{n}} \\
& \leq \frac{\lambda x_{n}}{1+\frac{\lambda-1}{k} x_{n}} .
\end{aligned}
$$

Next, we consider the following first-order difference equation

$$
u_{n+1}=\frac{\lambda u_{n}}{1+\frac{\lambda-1}{k} u_{n}} .
$$

Then, solution of (A.1) is given by:

$$
u_{n}=\frac{k}{1+\left(\frac{1}{\lambda}\right)^{n}\left(c_{1} k \lambda-1\right)}
$$


where $c_{1}$ depends upon initial value $u_{0}$ of difference equation (A.1). Since $\lambda>1$, therefore obviously one has

$$
\lim _{n \rightarrow \infty} u_{n}=k .
$$

Suppose that $x_{0}=u_{0}$, then by comparison we have $\lim _{n \rightarrow \infty} \sup x_{n} \leq k$ for all $n \geq 0$. It is easy to see that second equation of system (1.2) gives $y_{n+1} \leq b x_{n}$, and thus it follows that $\lim _{n \rightarrow \infty} \sup y_{n} \leq b k$. Again, we consider first equation of the system (1.2) as follows:

$$
\begin{aligned}
x_{n+1} & =\frac{\lambda x_{n}}{\exp \left(\frac{a y_{n}}{1+h x_{n}}\right)+\frac{\lambda-1}{k} x_{n}} \\
& \geq \frac{\lambda x_{n}}{\exp \left(\frac{a b k}{1+h x_{n}}\right)+\frac{\lambda-1}{k} x_{n}} \\
& \geq \frac{\lambda x_{n}}{e^{a b k}+\frac{\lambda-1}{k} x_{n}} .
\end{aligned}
$$

Assume that $\lambda>e^{a b k}$, then again comparison argument yields that $\lim _{n \rightarrow \infty} \inf x_{n} \geq m_{1}$, where

$$
m_{1}:=\frac{k\left(\lambda-e^{a b k}\right)}{\lambda-1}
$$

Keeping in view the inequality $\frac{x}{1+x} \leq\left(1-e^{-x}\right)$ and considering second equation of the system (1.2), we have

$$
y_{n+1} \geq \frac{a b m_{1} y_{n}}{1+h k+a y_{n}} .
$$

Finally, we assume that $a b m_{1}>1+h k$, then with comparison argument one has $\lim _{n \rightarrow \infty} \inf y_{n} \geq m_{2}$, where

$$
m_{2}:=\frac{a b m_{1}-(1+h k)}{a}
$$

Acknowledgements. The authors thank the main editor and anonymous referees for their valuable comments and suggestions leading to improvement of this paper.

\section{REFERENCES}

[1] E.H. Abed, H.O. Wang and R.C. Chen, Stabilization of period-doubling bifurcation and implications for control of chaos. Physica D $\mathbf{7 0}$ (1994) 154-164.

[2] F. Barraquand, S. Louca, K.C. Abbott, et al., Moving forward in circles: challenges and opportunities in modelling population cycles. Ecol. Lett. 20 (2017) 1074-1092.

[3] V.A. Bailey and A.J. Nicholson, The balance of animal populations. Proc. Zool. Soc. Lond. 3 (1935) 551-598.

[4] E. Bešo, S. Kalabušić, N. Mujić and E. Pilav, Neimark-Sacker bifurcation and stability of a certain class of a host-parasitoid models with a host refuge effect. Int. J. Bifurcat. Chaos 29 (2019) 195169.

[5] E. Bešo, N. Mujić, S. Kalabušić, E. Pilav, Stability of a certain class of a host-parasitoid models with a spatial refuge effect. J. Biol. Dyn. 14 (2020) 1-31.

[6] J.R. Beddington, C.A. Free and J.H. Lawton, Dynamic complexity in predator-prey models framed in difference equations. Nature 225 (1975) 58-60.

[7] M. Begon, C. Townsend, L. John, R. Colin and L.H. John, Ecology: From Individuals to Ecosystems. Oxford, Blackwell Publishing Ltd (2006).

[8] F. Brauer and C. Castillo-Chavez, Mathematical Models in Population Biology and Epidemiology. New York, Springer (2012). 
[9] F. Brauer, Z. Feng and C. Castillo-Chavez, Discrete epidemic models. Math. Biosci. Eng. 7 (2010) 1-15.

[10] J. Carr, Application of Center Manifold Theory. Springer-Verlag, New York (1981).

[11] G. Chen, J. Fang, Y. Hong and H. Qin, Controlling Hopf bifurcations: discrete-time systems. Discrete Dyn. Nat. Soc. 5 (2000) 29-33.

[12] G. Chen and X. Yu, On time-delayed feedback control of chaotic systems. IEEE Trans. Circ. Sys. 46 (1999) $767-772$.

[13] U. Daugaard, O.L. Petchey and F. Pennekamp, Warming can destabilise predator - prey interactions by shifting the functional response from Type III to Type II. J. Anim. Ecol. 88 (2019) 1575-1586.

[14] J.P. DeLong, T.C. Hanley and D.A. Vasseur, Predator-prey dynamics and the plasticity of predator body size. Funct. Ecol. 28 (2014) 487-493.

[15] Q. Din, Complexity and chaos control in a discrete-time prey-predator model. Commun. Nonlinear Sci. Numer. Simul. 49 (2017) 113-134.

[16] Q. Din, Stability, Bifurcation analysis and chaos control for a predator-prey system. J. Vib. Control 25 (2019) $612-626$.

[17] Q. Din, A novel chaos control strategy for discrete-time Brusselator models. J. Math. Chem. 56 (2018) 3045-3075.

[18] M. Fan and K. Wang, Periodic solutions of a discrete time nonautonomous ratio-dependent predator-prey system. Math. Comput. Model. 35 (2002) 951-961.

[19] J. Guckenheimer and P. Holmes, Nonlinear Oscillations, Dynamical Systems, and Bifurcations of Vector Fields. SpringerVerlag, New York (1983).

[20] K.P. Hadeler and I. Gerstmann, The discrete Rosenzweig model. Math. Biosci. 98 (1990) 49-72.

[21] A. Hastings, Population Biology: Concepts and Models. Springer, New York (1997).

[22] M.P. Hassell, The dynamics of arthropod predator - prey systems. Princeton University Press, Princeton (1978).

[23] M.P. Hassell and G.C. Varley, New inductive population model for insect parasites and its bearing on biological control. Nature 223 (1969) 1133-1137.

[24] J. Hofbauer, V. Hutson and W. Jansen, Coexistence for systems governed by difference equations of Lotka-Volterra type. $J$. Math. Biol. 25 (1987) 553-570.

[25] W.T. Jamieson, On the global behaviour of May's host-parasitoid model. J. Differ. Equ. Appl. 25 (2019) 583-596.

[26] V. Kaitala, J. Ylikarjula, M. Heino, Chaos in functional response host-parasitoid ecosystem models. Chaos Soliton Fract. 197 (1999) 331-341.

[27] B.E. Kendall, C.J. Briggs, W.W. Murdoch et al., Why do populations cycle? A synthesis of statistical and mechanistic modeling approaches. Ecology 80 (1999) 1789-1805.

[28] Y.A. Kuznetsov, Elements of Applied Bifurcation Theory. Springer-Verlag, New York (1997).

[29] V. Křivan and A. Priyadarshi, L-shaped prey isocline in the Gause predator-prey experiments with a prey refuge. J. Theor. Biol. 370 (2015) 21-26.

[30] H. Liu, K. Zhang, Y. Ye, Y. Wei and M. Ma, Dynamic complexity and bifurcation analysis of a host-parasitoid model with Allee effect and Holling type III functional response. Adv. Differ. Equ. 2019 (2019) 507.

[31] X. Liu, Y. Chu and Y. Liu, Bifurcation and chaos in a host-parasitoid model with a lower bound for the host. Adv. Differ. Equ. 2018 (2018) 31.

[32] P. Liu and S.N. Elaydi, Discrete competitive and cooperative models of Lotka-Volterra type. J. Comput. Anal. Appl. 3 (2001) $53-73$.

[33] X. Liu and D. Xiao, Complex dynamic behaviors of a discrete-time predator-prey system. Chaos Soliton Fract. 32 (2007) $80-94$.

[34] S.J. Lv and M. Zhao, The dynamic complexity of a host-parasitoid model with a lower bound for the host. Chaos Soliton Fract. 36 (2008) 911-919.

[35] X.S. Luo, G.R. Chen, B.H. Wang, and J.Q. Fang, Hybrid control of period-doubling bifurcation and chaos in discrete nonlinear dynamical systems. Chaos Soliton Fract. 18 (2003) 775-783.

[36] R.M. May, Host-parasitoid systems in patchy environments: a phenomenological model. J. Animal Ecol. 47 (1978) 833-844.

[37] R.M. May, M.P. Hassell, R.M. Anderson and D.W. Tonkyn, Density dependence in host-parasitoid models. J. Animal Ecol. 50 (1981) 855-865.

[38] W.W. Murdoch, C.J. Briggs and R.M. Nisbet, Consumer-Resource Dynamics. Princeton and Oxford, Princeton University Press (2003).

[39] M. Neubert and M. Kot, The subcritical collapse of predator populations in discrete time predator-prey models. Math. Biosci. 110 (1992) 45-66.

[40] K. Ogata, Modern Control Engineering, 2nd edition. Prentice-Hall, Englewood, New Jersey (1997).

[41] E. Ott, C. Grebogi and J.A. Yorke, Controlling chaos. Phys. Rev. Lett. 64 (1990) 1196-1199.

[42] J.L. Ren and L.P. Yu, Codimension-Two Bifurcation, Chaos and control in a discrete-time information diffusion model. $J$. Nonlinear Sci. 26 (2016) 1895-1931.

[43] C. Robinson, Dynamical Systems: Stability, Symbolic Dynamics and Chaos. Boca Raton, New York (1999).

[44] F.J. Romeiras, C. Grebogi, E. Ott and W.P. Dayawansa, Controlling chaotic dynamical systems. Physica D 58 (1992) $165-192$.

[45] B. Rosenbaum and B.C. Rall, Fitting functional responses: direct parameter estimation by simulating differential equations. Methods Ecol. Evol. 9 (2018) 2076-2090.

[46] M.L. Rosenzweig and R.H. MacArthur, Graphical representation and stability conditions of predator-prey interactions. Am. Nat. 97 (1963) 209-223. 
[47] P.S. Salomon and W. Stolte, Predicting the population dynamics in Amoebophrya parasitoids and their dinoflagellate hosts using a mathematical model. Mar. Ecol. Prog. Ser. 419 (2010) 1-10.

[48] S.Y. Tang and L.S. Chen, Chaos in functional response host-parasitoid ecosystem models. Chaos Soliton Fract. 13 (2002) $875-884$.

[49] J.T. Tanner, The stability and the intrinsic growth rates of prey and predator populations. Ecology 56 (1975) 855-867.

[50] A.D. Taylor, Parasitiod competition and the dynamics of host-parasitiod models. Am. Nat. 132 (1988) $417-436$.

[51] P. Turchin, Complex Population Dynamics. A Theoretical/Empirical Synthesis. Princeton, Princeton University Press (2003).

[52] E. van Velzen and U. Gaedke, Reversed predator-prey cycles are driven by the amplitude of prey oscillations. Ecol. Evol. 8 (2018) 6317-6329.

[53] Y.H. Wan, Computation of the stability condition for the Hopf bifurcation of diffeomorphism on $R^{2}$. SIAM.J. Appl. Math. 34 (1978) 167-175.

[54] G.L. Wen, D.L. Xu, and J.H. Xie, Controlling Hopf bifurcations of discrete-time systems in resonance. Chaos Soliton Fract. 23 (2005) 1865-1877.

[55] W. Wendi and L. Zhengyi, Global stability of discrete models of Lotka-Volterra type. Nonlinear Anal. Theor. 35 (1999) 1019-1030.

[56] V. Weide, M.C. Varriale and F.M. Hilker, Hydra effect and paradox of enrichment in discrete-time predator-prey models. Math. Biosci. 310 (2019) 120-127.

[57] S. Wiggins, Introduction to Applied Nonlinear Dynamical Systems and Chaos. Springer-Verlag, New York (2003).

[58] D. Wu and H. Zhao, Global qualitative analysis of a discrete host-parasitoid model with refuge and strong Allee effects. Math. Method. Appl. Sci. 41 (2018) 2039-2062.

[59] C.L. Xu and M.S. Boyce, Dynamic complexities in a mutual interference host-parasitoid model. Chaos Soliton Fract. 24 (2005) $175-182$.

[60] L.-G. Yuan and Q.-G. Yang, Bifurcation, invariant curve and hybrid control in a discrete-time predator-prey system. Appl. Math. Model. 39 (2015) 2345-2362.

[61] X. Zhang, Q.L. Zhang and V. Sreeram, Bifurcation analysis and control of a discrete harvested prey-predator system with Beddington-DeAngelis functional response. J. Franklin Inst. 347 (2010) 1076-1096.

[62] L. Zhu and M. Zhao, Dynamic complexity of a host-parasitoid ecological model with the Hassell growth function for the host. Chaos Soliton Fract. 39 (2009) 1259-1269. 